\title{
Statistical Topology of Three-Dimensional Poisson-Voronoi Cells and Cell Boundary Networks
}

\author{
Emanuel A. Lazar ${ }^{1,5}$, Jeremy K. Mason ${ }^{2,3}$, Robert D. MacPherson ${ }^{4}$, David J. Srolovitz ${ }^{5}$ \\ ${ }^{1}$ Applied Physics and Applied Mathematics, Columbia University, New York, New York 10027. \\ ${ }^{2}$ Lawrence Livermore National Laboratory, Livermore, California 94550. \\ ${ }^{3}$ Boğaziçi University, Bebek, Istanbul 34342 Türkiye. \\ ${ }^{4}$ School of Mathematics, Institute for Advanced Study, Princeton, New Jersey 08540. \\ ${ }^{5}$ Materials Science and Engineering, University of Pennsylvania, Philadelphia, PA 19104.
}

(Dated: August 31, 2018)

\begin{abstract}
Voronoi tessellations of Poisson point processes are widely used for modeling many types of physical and biological systems. In this paper, we analyze simulated Poisson-Voronoi structures containing a total of 250,000,000 cells to provide topological and geometrical statistics of this important class of networks. We also report correlations between some of these topological and geometrical measures. Using these results, we are able to corroborate several conjectures regarding the properties of three-dimensional Poisson-Voronoi networks and refute others. In many cases, we provide accurate fits to these data to aid further analysis. We also demonstrate that topological measures represent powerful tools for describing cellular networks and for distinguishing among different types of networks.
\end{abstract}

PACS numbers: 61.72.-y, 61.43.Bn, 05.10.-a

\section{INTRODUCTION}

Poisson-Voronoi tessellations are random subdivisions of space that have found applications as models for many physical systems [1, 2]. They have been used to study how galaxies are distributed throughout space [3, 4] and have aided in discovering new galaxies [5]. They have been used to study how animals establish territories [6], how crops can be planted to minimize weed growth 7], and how atoms are arranged in crystals [8], liquids [9], and glasses [10, 11]. A more complete list of applications can be found in standard references on the subject [1, 2].

A Poisson-Voronoi tessellation is constructed as follows. Points called seeds are obtained as the realization of a uniform Poisson point process (e.g. see 2, 12 15]) in a fixed region. Cells are the sets of all points in the region that are closer to a particular seed than to any other. If a point is equidistant to multiple nearest seeds, then it lies on the boundary of the associated cells. In three dimensions, there is a zero probability that a point will be equidistant to five or more seeds. All cells are convex, and this network of cells partitions the entire region.

Many exact results have been obtained in connection with three-dimensional Poisson-Voronoi structures. Meijering [16] proved that the average number of faces per cell is $48 \pi^{2} / 35+2 \approx 15.535$, the average number of edges per face is $144 \pi^{2} /\left(35+24 \pi^{2}\right) \approx 5.228$, the average surface area per cell is $(256 \pi / 3)^{1 / 3} \Gamma\left(\frac{5}{3}\right) \rho^{-2 / 3}$ and the average edge length per cell is $\left(3072 \pi^{5} / 125\right)^{1 / 3} \Gamma\left(\frac{4}{3}\right) \rho^{-1 / 3}$, where $\rho$ is the number of seeds or cells per unit volume. Gilbert [17] expressed the variance of the cell volume distribution as a double integral. Using a more general approach, Brakke [18] obtained integral expressions for the variances of number of cell faces, volumes, surface areas, number of face edges, face areas, and perimeters, as well as variances and covariances of several other quantities of interest and the distribution of edge lengths. In all of these cases, Brakke also solved these integrals numerically. Much more is understood about Poisson-Voronoi structures than can be detailed here, and the interested reader is referred to standard references [1, 2, 19] and the more recent surveys of Møller and Stoyan [20] and of Calka in [21].

Additional properties of Poisson-Voronoi structures have been investigated through simulation. Using a data set with 358,000 cells, Kumar et al. 22] reported the distributions of faces with fixed numbers of edges and cells with fixed numbers of faces, volumes, face areas and cell surface areas. They also reported distributions of volumes and surface areas restricted to cells with fixed numbers of faces, and distributions of areas and perimeters restricted to faces with fixed numbers of edges. Although their data set was relatively small by current standards, their results are the most complete set of threedimensional Poisson-Voronoi cell statistics available in the literature.

The Kumar et al. data set has since been augmented by additional results. Marthinsen 23] used a set of 100,000 cells to compute the distribution of cell volumes and surface areas. Tanemura [24] later used a substantially larger data set of 5,000,000 cells to obtain more precise data for the distributions of volumes, surfaces areas, and faces, as well as volumes for cells with fixed numbers of faces. Ferenc and Néda [25] later used a data set with $18,000,000$ cells to calculate the distribution of cell volumes.

Thorvaldsen [26] and Reis et al. 227] used the ratio between the surface area of a cell and the surface area of a sphere of equal volume to describe the "shape isotropy" of a cell. Using a system of 250,000 cells, Thorvaldsen reported the distribution of this parameter among Poisson-Voronoi cells, and observed that this parameter 
decreases with increasing cell volume. Using a smaller set of 10,000 cells, Reis et al. considered how this parameter depends on the number of faces of a cell. A more sophisticated, higher-order method of measuring shape isotropy using Minkowski tensors has been recently introduced [28] and used to characterize a number of natural structures [29, 30]. In particular, Kapfer et al. [29] have used this method to characterize a data set of 160,000 Poisson-Voronoi cells [29].

In prior studies, the topology of individual cells has been described by counting their numbers of faces. As we discuss below, this is a simplistic and incomplete description of the topology of a cell] In this report, we present distributions of many important topological features of Poisson-Voronoi structures based on a dataset of a combined total of $250,000,000$ cells. This is the largest data set available today and provides the most precise characterization of topological properties of the PoissonVoronoi network. This resolution allows us to examine the validity of conjectures made on the basis of smaller data sets, some of which we now show are qualitatively incorrect. We supplement the discussion of topological properties with analysis of some purely geometrical descriptions and the interrelationship between some topological and geometrical features. We leave many results in the Supplemental Material and make the entire data set available online 31.

\section{METHOD}

We employ the computer code vor3dsim, developed by Ken Brakke [32], to generate 250 Poisson-Voronoi tessellations, each of which contains 1,000,000 cells; periodic boundary conditions are used to eliminate boundary effects. Because the statistics we consider measure neighborhoods of the structure which are small compared to the total size of the system, we expect that statistics observed in this set of smaller systems will be consistent with what we would observe in a single system with an identical number of total cells. Details of the algorithms used to perform some of the more complex topological analyses were reported previously [33, 34].

\section{TOPOLOGICAL CHARACTERISTICS}

\section{A. Distribution of faces}

The simplest way to classify the topology of a PoissonVoronoi cell involves counting its number of faces. This is the topological characterization most commonly quoted

\footnotetext{
${ }^{1}$ When referring to the topology of a cell we have in mind the topology of the cell and its immediate neighborhood, which includes the network of edges and faces which intersect it.
}

in the literature [35, 36]. Figure 1 shows this distribution of faces per cell; these data are consistent with those reported in 22] and 24]. As noted earlier, Meijering [16] proved that the mean of this distribution is $48 \pi^{2} / 35+2$. Brakke [18] obtained an integral form of the variance, which he numerically evaluated to be 11.1246 . Our data reproduce these exact results to within $0.001 \%$ and $0.004 \%$, respectively. While the distribution of faces is approximately symmetric about 15 , there are no cells with fewer than 4 faces. We note that while Kumar et al. 22] and Tanemura 24] reported no cells with more than 36 faces based on their more limited data set, we find cells with up to 41 faces.

Kumar et al. 22] suggested that the distribution of the number of faces $F$ per cell can be described by the discretized two-parameter $\Gamma$ function:

$$
p(F)=\int_{F-1 / 2}^{F+1 / 2} \frac{x^{a-1}}{b^{a} \Gamma(a)} e^{-x / b} d x .
$$

The best fit to their data yielded $a=21.6292$ and $b=0.7199$. The form of $p(F)$ in Eq. (1) gives $p(F)>0$ for all positive integers $F$, including 1,2 and 3. Of course, this is incorrect since there can be no polyhedra with fewer than four faces in a Voronoi tessellation. Moreover, since we know exactly both the mean of the distribution as well as its variance, we are left with no free parameters. Regardless of whether we choose to include $p(1), p(2)$, and $p(3)$ in normalizing the distribution, these parameters must be $a=21.85892$ and $b=0.710714$ to match the exact results. Careful inspection of the data in the inset to Fig. 1 reveals that Eq. (1) does not accurately describe the decay in $p(F)$ for large $F$. This further demonstrates that this conjectured equation is not an exact representation of $p(F)$; we know of no such exact relation.

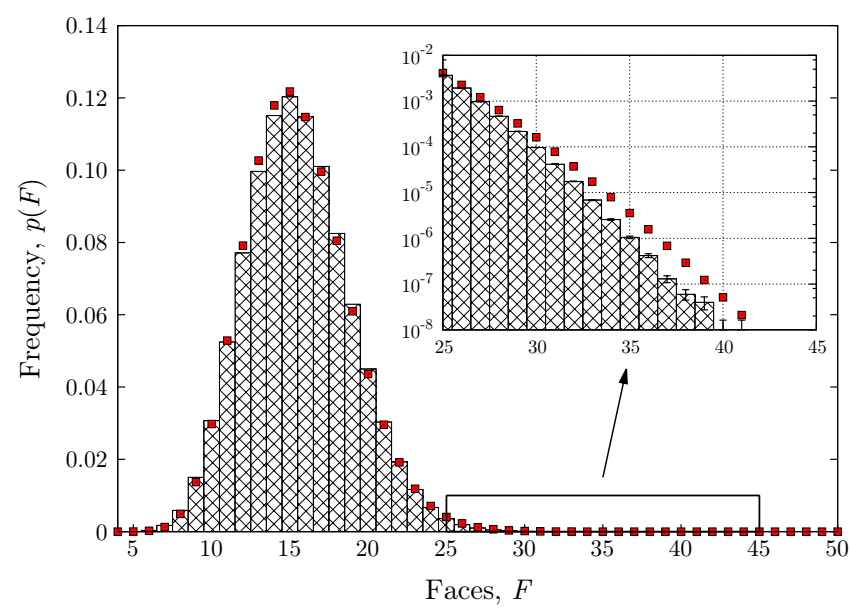

FIG. 1. Distribution of the number of faces per cell; squares show the discrete probability distribution of Eq. (1). The mean and standard deviation are 15.535 and 3.335, respectively, to within the accuracy of the data. The inset shows a subset of the data on a semilogarithmic plot; error bars show the standard error from the mean. 


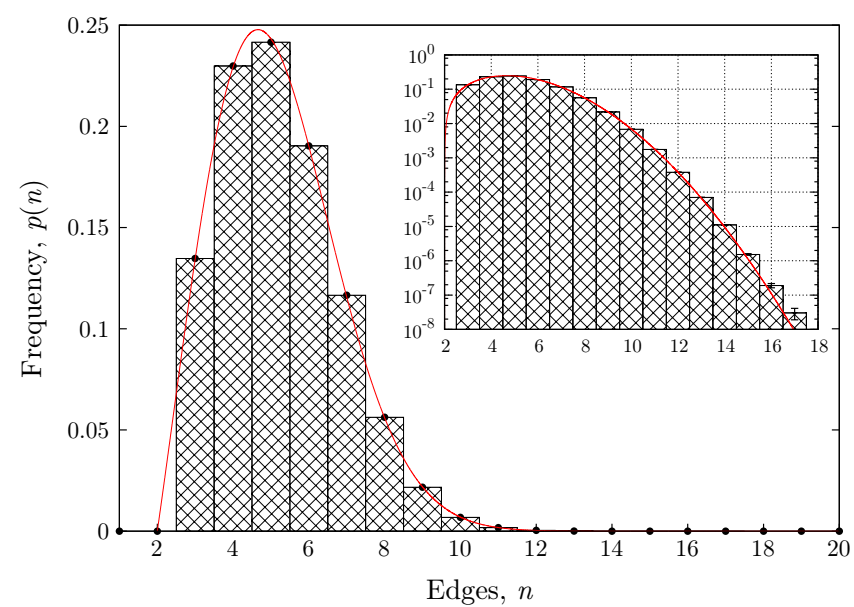

FIG. 2. Distribution of number of edges per face; squares show the discrete probability distribution of Eq. (2). The mean and standard deviation for this data set is 5.228 and 1.576, respectively, to within the accuracy of the data. The inset shows the same data on a semilogarithmic plot; error bars show the standard error from the mean.

\section{B. Distribution of edges}

We next consider the distribution $p(n)$ of faces with $n$ edges. Meijering [16] proved that the mean of this distribution is $144 \pi^{2} /\left(35+24 \pi^{2}\right)$, and Brakke [18] obtained an integral form of the variance, which he evaluated numerically to be 2.4846 . Our data reproduce the exact result of the mean to within $0.0002 \%$, and the exact result for the variance to within $0.00001 \%$. Figure 2 shows this distribution for our Poisson-Voronoi data set. This distribution is similar to that reported earlier [22], albeit with more accurate statistics and over a greater range of $n$. The distribution has a maximum at 5 edges per face, which is close to the mean; there are no faces with fewer than 3 edges. While Kumar et al. 22] reported no faces with more than 15 edges, our data set shows faces with up to 18 edges.

While the mean and variance of this distribution are known exactly, little else is known and, to our knowledge, there are no proposed forms for this distribution. We suggest the following empirical form

$$
p(n)= \begin{cases}A(n-2) e^{-B(n-\hat{n})^{2}} & n \geq 2 \\ 0 & n<2,\end{cases}
$$

where $n$ is the number of edges of a face. Although this empirical relation fits the data remarkably well, its origin is unclear. By requiring that the distribution is properly normalized and that the mean and variance reproduce the exact results, all three parameters are determined: $A=0.13608070, B=0.093483172$, and $\hat{n}=2.64631320$. Overall, this empirical functional form provides an excellent fit to the Poisson-Voronoi data set, including the large $n$ tail.

\section{Aboav-Weaire relation}

In studying two-dimensional cross-sections of polycrystalline magnesium oxide, Aboav [37] and Weaire [38] explored the relationship between the number of edges $n$ of a cell and the expected number of edges $m(n)$ of its $n$ neighbors. They observed that this relationship can be described by $m(n) \approx A+B / n$, which can be understood as follows. In two dimensions, the average number of edges per cell is $\langle n\rangle=6$. If this average is approximately maintained among every cluster of cells, then a cluster with an $n$-sided cell in its center should have on average $(n+n m(n)) /(n+1) \approx\langle n\rangle$ edges per cell. This gives us an expression for $m(n)$ in terms of $n$ and $\langle n\rangle$ of the above form: $m(n) \approx 5+6 / n$. This equivalence is only approximate because the $\langle n\rangle=6$ average is not maintained among every cluster of cells. This leads to a correction term that in part depends on the variance of the distribution. We note, for later, that this form of $m(n)$ decreases monotonically with increasing $n$. This relationship has been used to analyze biological tissue [39, 40], soap foams [41, 42], and other cellular structures [43 45].

In two dimensions, it was originally believed that this relationship also describes Poisson-Voronoi structures [46, 47]. However, Hilhorst [48, 49] has shown that the correct form of the relationship is $m(n)=$ $4+3(\pi / n)^{1 / 2}+O(1 / n)$, in the limit of large $n$.

We now investigate the extension of this relationship to three-dimensional Poisson-Voronoi structures, i.e., the relationship between the number of faces $F$ of a cell and the expected number of faces $m(F)$ of its neighbors. Figure 3 shows that $m(F)$ increases for small $F$, reaches a maximum at $F=12$, and then decreases in a nearly linear manner for large $F$. The existence of the increasing region of $m(F)$ has not been previously reported for the Poisson-Voronoi structure.

Based on limited three-dimensional Poisson-Voronoi data (3729 cells), Kumar et al. 22] fit their data to a linear function as follows:

$$
m(F)=A-B F,
$$

and found $A=16.57$ and $B=0.02$. Of course, such a fit is unreasonable because it suggests that $m(F)<0$ for sufficiently large $F$. Using the same data set as Kumar et al., Fortes [50] proposed fitting this data to an AboavWeaire-type of relation:

$$
m(F)=A+B / F,
$$

where the constants $A=15.96$ and $B=4.60$ were found using a least square fit to this data set. The forms suggested by Kumar et al. and Fortes do not provide even a qualitative fit to $m(F)$ at small $F$; they both decrease monotonically with increasing $F$, contrary to the data for $F<12$. Clearly, the general form of the Aboav-Weaire relation provides a poor representation of the topological correlations between nearest neighbor cells in threedimensional Poisson-Voronoi structures. 


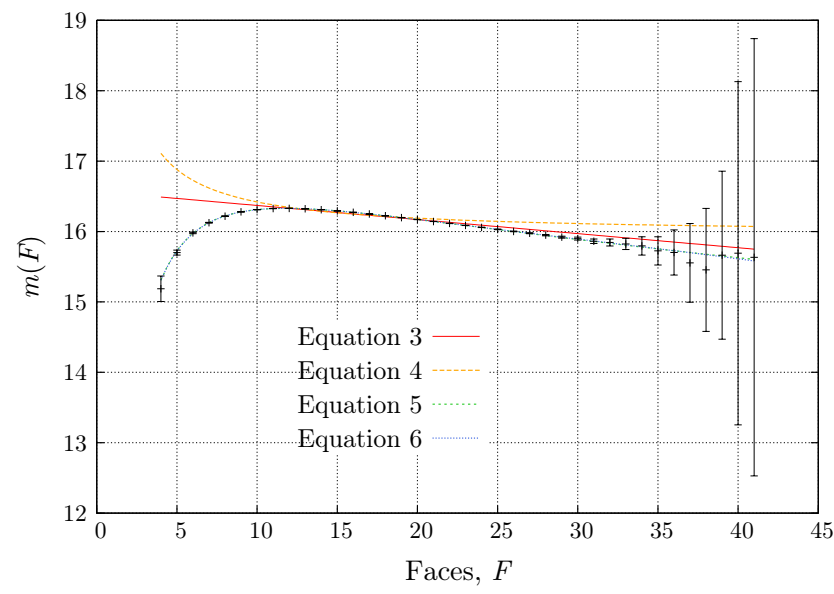

FIG. 3. (Color online) Expected number of faces $m(F)$ of neighbors of cells with $F$ faces; error bars indicate standard error from the mean. The red, yellow, green, and blue curves show the forms suggested by Kumar et al., Fortes, Hillhorst (truncated at $i=3$ ), and Mason et al. (truncated at $i=4$ ), respectively.

Hilhorst [51], building on his earlier work on twodimensional Poisson-Voronoi structures [48, 49], provided strong theoretical arguments for a relationship of the form:

$$
m(F)=k_{0}+\sum_{i=1}^{\infty} k_{i} F^{-i / 6}
$$

with $k_{0}=8$. A least squares fit of this expression (truncated at $i=3$ ) to our data yields $k_{1}=2.474, k_{2}=49.36$, and $k_{3}=-51.50$. This form provides an excellent fit to the present three-dimensional Poisson-Voronoi data set, over the entire range of $F$.

Finally, Mason et al. 52], also based on theoretical arguments, developed the following expression for $m(F)$ :

$m(F)=\langle F\rangle+\frac{\langle F\rangle+\mu_{2}}{F}-1-\frac{1}{\xi F} \sum_{i=1}^{\infty} k_{i}\left[(F-\langle F\rangle)^{i}-\mu_{i}\right]$,

where $\xi=4 \pi-6 \cos ^{-1}(-1 / 3)$ is a constant for threedimensional structures, $\mu_{i}$ is the $i^{\text {th }}$ central moment of the distribution of faces, and $k_{i}$ are fitting parameters. The equation shown in Fig. 3 corresponds to a best fit when considering $i \leq 4$; we find that $k_{1}=-1.567, k_{2}=$ $0.0478, k_{3}=-0.00109$, and $k_{4}=0.000022$. Except at the tail end of $m(F)$, Eqs. (5) and (6) are indistinguishable.

\section{D. $p$-vectors}

Although counting faces can often distinguish between topologically distinct cells, it cannot do so in general. Figure 4 shows topologically distinct cells, each with six faces. A more refined description of the topology of a cell

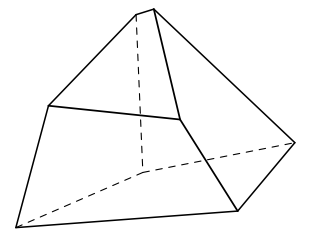

(a)

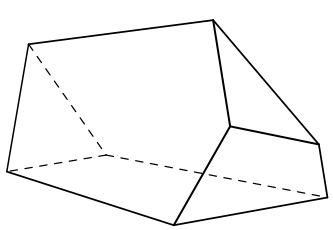

(b)
FIG. 4. Topologically distinct cells with six faces. Type (a) appears more than twice as frequently as type (b) in the Poisson-Voronoi structure.

involves recording not only its number of faces, but also its particular types of faces. Figure 4 (a) has six foursided faces, while Fig. 4(b) has two three-sided faces, two four-sided faces, and two five-sided faces. These two topological types are the only ones with six faces that appear in the Poisson-Voronoi structures.

Barnette [53], in describing the combinatorial properties of three-dimensional polytopes, defined a $p$-vector as a vector of integer entries in which the $i^{\text {th }}$ entry denotes the number of $i$-sided faces of a polyhedron. Table Ilists the 48 most frequent $p$-vectors of the Poisson-Voronoi structure and their relative frequencies in the PoissonVoronoi dataset. The table shows that the PoissonVoronoi structure is not dominated by a small set of $p$-vectors; rather, the distribution is quite broad - no $p$-vector occurs with a frequency greater than $0.39 \%$. In the 250,000,000 Poisson-Voronoi cell data set, there are 375,410 distinct $p$-vectors; the complete distribution of observed $p$-vectors may be found in the Supplemental Material.

Poisson-Voronoi cells may be contrasted with those found in other natural structures. Matzke [54] carefully recorded $p$-vector data for 1000 soap bubbles in a foam, and Williams and Smith 55] reported $p$-vector data for 91 individual cells in an aluminum polycrystal. More recently, Kraynik et al. [56] reported $p$-vector data to characterize over 1000 simulated monodisperse foam bubbles, and we have reported the distribution of $p$-vectors in a set of 269,555 grains in simulated grain growth microstructures [33].

Although soap foams and grain growth microstructures share much in common with Poisson-Voronoi tessellations, it is important to emphasize that they result from qualitatively different processes. Capillarity, surface tension, and curvature play significant roles in the formation and evolution of soap foams [41] and grain growth microstructures [57]. Since these forces tend to minimize interfacial areas (subject to certain constraints), we can expect that the microstructures that result from these processes somehow reflect these physics. More specifically, we might expect to find qualitatively different microstructures than those that result from a PoissonVoronoi tessellation, in which these forces play no role.

The data reported in [54], [55], and [56] are insufficient to provide definitive $p$-vector distributions for either polycrystalline aluminum or soap foam structures. 


\begin{tabular}{|r|c|r|c|}
\hline & $p$-vector & $F$ & $f$ \\
\hline 1 & $(001343100 \ldots)$ & 12 & $0.388 \%$ \\
2 & $(001342100 \ldots)$ & 11 & $0.342 \%$ \\
3 & $(001433200 \ldots)$ & 13 & $0.298 \%$ \\
4 & $(001344100 \ldots)$ & 13 & $0.289 \%$ \\
5 & $(001423100 \ldots)$ & 11 & $0.288 \%$ \\
6 & $(002333110 \ldots)$ & 13 & $0.284 \%$ \\
7 & $(001332000 \ldots)$ & 9 & $0.274 \%$ \\
8 & $(000442000 \ldots)$ & 10 & $0.265 \%$ \\
9 & $(001352200 \ldots)$ & 13 & $0.263 \%$ \\
10 & $(002233100 \ldots)$ & 11 & $0.261 \%$ \\
11 & $(001432200 \ldots)$ & 12 & $0.258 \%$ \\
12 & $(001353200 \ldots)$ & 14 & $0.258 \%$ \\
\hline
\end{tabular}

\begin{tabular}{|c|c|c|c|}
\hline & $p$-vector & $F$ & $f$ \\
\hline 13 & $(002332110 \ldots)$ & 12 & $0.256 \%$ \\
14 & $(001422100 \ldots)$ & 10 & $0.254 \%$ \\
15 & $(002322200 \ldots)$ & 11 & $0.252 \%$ \\
16 & $(002242200 \ldots)$ & 12 & $0.248 \%$ \\
17 & $(002342210 \ldots)$ & 14 & $0.247 \%$ \\
18 & $(001443110 \ldots)$ & 14 & $0.244 \%$ \\
19 & $(000443000 \ldots)$ & 11 & $0.239 \%$ \\
20 & $(002343210 \ldots)$ & 15 & $0.233 \%$ \\
21 & $(001442110 \ldots)$ & 13 & $0.232 \%$ \\
22 & $(001424100 \ldots)$ & 12 & $0.231 \%$ \\
23 & $(001434200 \ldots)$ & 14 & $0.223 \%$ \\
24 & $(002243200 \ldots)$ & 13 & $0.217 \%$ \\
\hline
\end{tabular}

\begin{tabular}{|c|c|c|c|}
\hline & $p$-vector & $F$ & $f$ \\
\hline 25 & $(002323200 \ldots)$ & 12 & $0.213 \%$ \\
26 & $(002232100 \ldots)$ & 10 & $0.210 \%$ \\
27 & $(002423210 \ldots)$ & 14 & $0.203 \%$ \\
28 & $(002334110 \ldots)$ & 14 & $0.202 \%$ \\
29 & $(001252000 \ldots)$ & 10 & $0.201 \%$ \\
30 & $(000533100 \ldots)$ & 12 & $0.199 \%$ \\
31 & $(001263100 \ldots)$ & 13 & $0.198 \%$ \\
32 & $(001341100 \ldots)$ & 10 & $0.196 \%$ \\
33 & $(002234100 \ldots)$ & 12 & $0.193 \%$ \\
34 & $(001354200 \ldots)$ & 15 & $0.192 \%$ \\
35 & $(001345100 \ldots)$ & 14 & $0.191 \%$ \\
36 & $(001334000 \ldots)$ & 11 & $0.190 \%$ \\
\hline
\end{tabular}

\begin{tabular}{|c|c|c|c|}
\hline & $p$-vector & $F$ & $f$ \\
\hline 37 & $(002422210 \ldots)$ & 13 & $0.190 \%$ \\
38 & $(002333300 \ldots)$ & 14 & $0.188 \%$ \\
39 & $(002324200 \ldots)$ & 13 & $0.188 \%$ \\
40 & $(001442300 \ldots)$ & 14 & $0.186 \%$ \\
41 & $(001444110 \ldots)$ & 15 & $0.185 \%$ \\
42 & $(001443300 \ldots)$ & 15 & $0.185 \%$ \\
43 & $(002332300 \ldots)$ & 13 & $0.180 \%$ \\
44 & $(001351200 \ldots)$ & 12 & $0.179 \%$ \\
45 & $(000453100 \ldots)$ & 13 & $0.178 \%$ \\
46 & $(001533210 \ldots)$ & 15 & $0.175 \%$ \\
47 & $(001333000 \ldots)$ & 10 & $0.173 \%$ \\
48 & $(001453210 \ldots)$ & 16 & $0.172 \%$ \\
\hline
\end{tabular}

TABLE I. The 48 most frequent $p$-vectors in the Poisson-Voronoi structure, their number of faces $F$ and their relative frequency $f$. The 250,000,000 cell data set contains 375,410 distinct $p$-vectors; the complete distribution of $p$-vectors may be found in the Supplemental Material.

However, they are sufficient to clearly distinguish those structures from the Poisson-Voronoi one. Of the 91 aluminum cells examined by Williams and Smith, the most common $p$-vector is $(0004420 \ldots)$, and it appeared 8 times. Seven other $p$-vectors appeared 2 or 3 times each, and the remaining 66 distinct $p$-vectors appeared only once each.

Considering only the interior bubbles of his original sample, Matzke found that the most common $p$-vector was $(0001102 \ldots)$. These bubbles accounted for $20 \%$ of the 600 interior bubbles. Three more $p$-vectors each accounted for at least $8 \%$ of all bubbles, five more accounted for at least $3 \%$ each, and five more accounted for at least $1.5 \%$ of all bubbles.

Kraynik et al. [56] found that data from simulated monodisperse foams closely resembled the experimental results of Matzke. In particular, they found that the most common $p$-vector was (0001 $102 \ldots)$, which accounted for just under $20 \%$ of all relaxed and annealed monodisperse foam bubbles, a result almost identical to that of Matzke. The next most common $p$-vector in simulated monodisperse structures was $(0002840 \ldots)$, which accounted for almost $14 \%$ of all bubbles, and then (0001 $103 \ldots)$, which accounted for just under $11 \%$ of all bubbles. Four other $p$-vectors each accounted for at least $5 \%$ of bubbles.

The distribution of $p$-vectors in grain growth structures 33], which evolve through mean curvature flow, is substantially more concentrated than in the PoissonVoronoi microstructure, but not nearly as much as in the data of Matzke, Williams and Smith, and Kraynik et al. In the grain growth data, the most common $p$-vector is $(0004400 \ldots)$, and it accounts for nearly $3 \%$ of all cells; each of the 10 most common $p$-vectors accounts for at least $1 \%$ of all cells.

Although the exact nature of the heavy bias towards certain $p$-vectors in each of the structures is not completely understood, it can already be used to distinguish the different structures from the Poisson-Voronoi structure and from each other by standard statistical tests (e.g., a chi-squared test). The distribution of $p$-vectors, hence, provides a useful means to distinguish between cellular structures of different physical origin. Despite its early introduction, this method has not been widely adopted.

\section{E. Distribution of topological types}

Although the $p$-vector of a cell provides more information than a mere count of its faces, it too does not completely describe its topology. For example, Fig. 5 shows three cells that share the $p$-vector $(0004420 \ldots)$ and yet are topologically distinct.

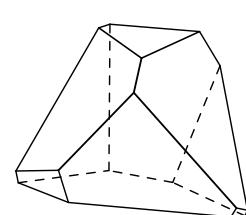

(a)

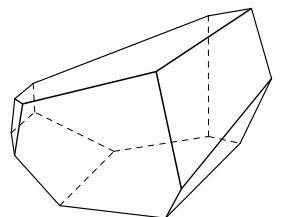

(b)

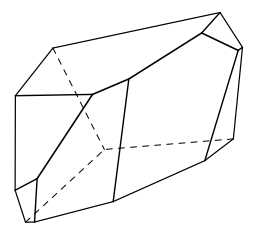

(c)
FIG. 5. Three topologically distinct cells, each with four quadrilateral, four pentagonal, and two hexagonal faces. Type (a) appears roughly twice as frequently as type (b), which appears roughly fives times as frequently as type (c), in the Poisson-Voronoi structure.

In an earlier paper [33], we developed a method to succinctly characterize the complete topology of a cell. That work was built on earlier work of Weinberg [58, 59], who developed an efficient graph-theoretic algorithm to determine whether two triply-connected planar graphs are isomorphic. We showed that the edges and vertices of a cell can be treated as a planar graph, and Weinberg's method can then be used to calculate what we call a Weinberg vector for each cell. A Weinberg vector for a cell with $F$ faces is a vector with $6(F-2)$ integer entries that can be computed in time linear in $F^{2}$. Two cells are topologically identical if and only if their Weinberg vectors are identical. Moreover, the method by which the Weinberg vector is calculated also determines the order of the cell's associated symmetry group 60]. The topological type of each cell in the structures is recorded, 
along with its $p$-vector, symmetry order, and frequency. We do not reproduce the algorithm for creating a Weinberg vector here, but simply refer the interested reader to 33 .

In the remainder of this paper, we use Schlegel diagrams to help visualize topological types. A Schlegel diagram is constructed by projecting the boundary of a cell onto one of its faces in a way that vertices not belonging to that face lie inside it and no edges cross 61, 62]. Figure 6 shows Schlegel diagrams for the 24 most common topological types that appear in the Poisson-Voronoi structure. Along with the Schlegel diagram for each, we show its frequency $f$, number of faces $F, p$-vector, and order $S$ of its symmetry group.

Each of the six most common topological types have 10 or fewer faces. This may be surprising in light of the fact that of the 48 most commonly occurring $p$-vectors, only one had fewer than 10 faces. This can be understood by considering that many distinct topological types can share the same $p$-vector, as illustrated earlier in Fig. 5. This degeneracy increases with the number of faces, and so $p$-vectors of cells with many faces can appear frequently even if no single topological type with that $p$-vector appears frequently. Conversely, $p$-vectors with few faces are typically shared by few distinct topological types. The most frequently occurring $p$-vector (001343100...) is shared by 38 distinct topological types 2 not one of which appears among the 24 most common types.

The most common topological type in the PoissonVoronoi structure (Fig. 6, entry 1) has p-vector (0013320...) and occurs with frequency $0.273 \%$. Two factors appear to contribute to its relative high frequency. First, its distribution of face types closely resembles that of the structure as a whole (Fig. 21). Specifically, fourand five-sided faces appear most frequently, followed by six-sided faces and then three-sided faces. Second, no other topological type shares this $p$-vector. Despite its frequency, however, it is difficult to describe it as a "typical" Poisson-Voronoi cell, given how few cells are of this type.

Figure 7 illustrates Schlegel diagrams of a number of highly symmetric polyhedra: the tetrahedron, truncated tetrahedron, cube, truncated cube, pentagonal dodecahedron, truncated pentagonal prism, pentagonal antiprism over a heptagon, and truncated octahedron. The first, third, and fifth of these are Platonic solids that occur with non-zero probability in the Poisson-Voronoi structures. The last of these shapes, often referred to as the Kelvin tetrakaidecaheron or Kelvin cell, was conjectured by Lord Kelvin [64, 65] to tile three-dimensional space with a minimal surface area, very much like the regular hexagon tiles the plane with minimal perimeter [66].

\footnotetext{
2 This can be extracted from data available on The Manifold Page 63]; data for these 38 types are included in Fig. 20 of the Supplemental Material.
}

It can be shown that every topological type appears in the Poisson-Voronoi tessellation with a non-zero frequency, and so the appearance of these highly symmetric shapes is not surprising. However, their relative frequencies warrant attention. The truncated cube and the Kelvin cell both have 14 faces and a symmetry group of order $S=48$, and yet they occur with substantially different frequencies. It is clear that frequencies are not entirely determined by the number of faces of a cell nor by the order of its associated symmetry group. It is unclear how these topological features impact frequency.

The 24 most common topological types in PoissonVoronoi structures account for less than $2.5 \%$ of all cells. By contrast, the distribution of topological types in grain growth structures is substantially more concentrated 33]. There, the 24 most common types account for over $25 \%$ of all cells [33]. While space-filling constraints in both the Poisson-Voronoi and grain growth structures create a bias towards certain topological types, the curvature flow process that governs the evolution of grain growth structures leads to a secondary bias towards cells that exhibit a low surface area to volume ratio. Distributions of topological types have not been collected, to the best of our knowledge, for other cellular structures.

\section{F. Order of symmetry groups}

As noted earlier, the algorithm which determines the Weinberg vector of a cell also determines the order of its associated symmetry group. Figure 8 shows the distribution of symmetry orders among all cells. Roughly $91.71 \%$ of cells have only the trivial symmetry (order 1), $6.61 \%$ have a symmetry of order 2 , and $1.00 \%$ have a symmetry of order 4 . The remaining $0.68 \%$ have symmetries of order 3 or higher than 4 . The probability of finding a cell with a particular symmetry order generally decreases quickly with the order, subject to certain secondary rules. More specifically, odd numbers and numbers whose prime factors are all large appear highly infrequently. Odd orders appear in topological types with rotational symmetries but without mirror or inversion symmetries. Therefore, we find no cells with symmetry order 13, for example, even though we find many with symmetry orders 16,24 and 48 .

The average symmetry order of Poisson-Voronoi cells is 1.16. This might be contrasted with the case of grain growth structures [67], where the average observed symmetry order is 3.09 [33]. This discrepancy may be due to the tendency of mean curvature flow to minimize surface area, although how this correlates with topological symmetry is unclear since curvature is a geometric quantity.

We note that although some symmetries of a cell can be observed in its Schlegel diagram, the diagram can often obscure other symmetries. Entries 5 and 21 in Fig. 6. for example, might appear at first sight more symmetric than entry 6 , and yet the latter has the highest symmetry order of the three. To understand this apparent 


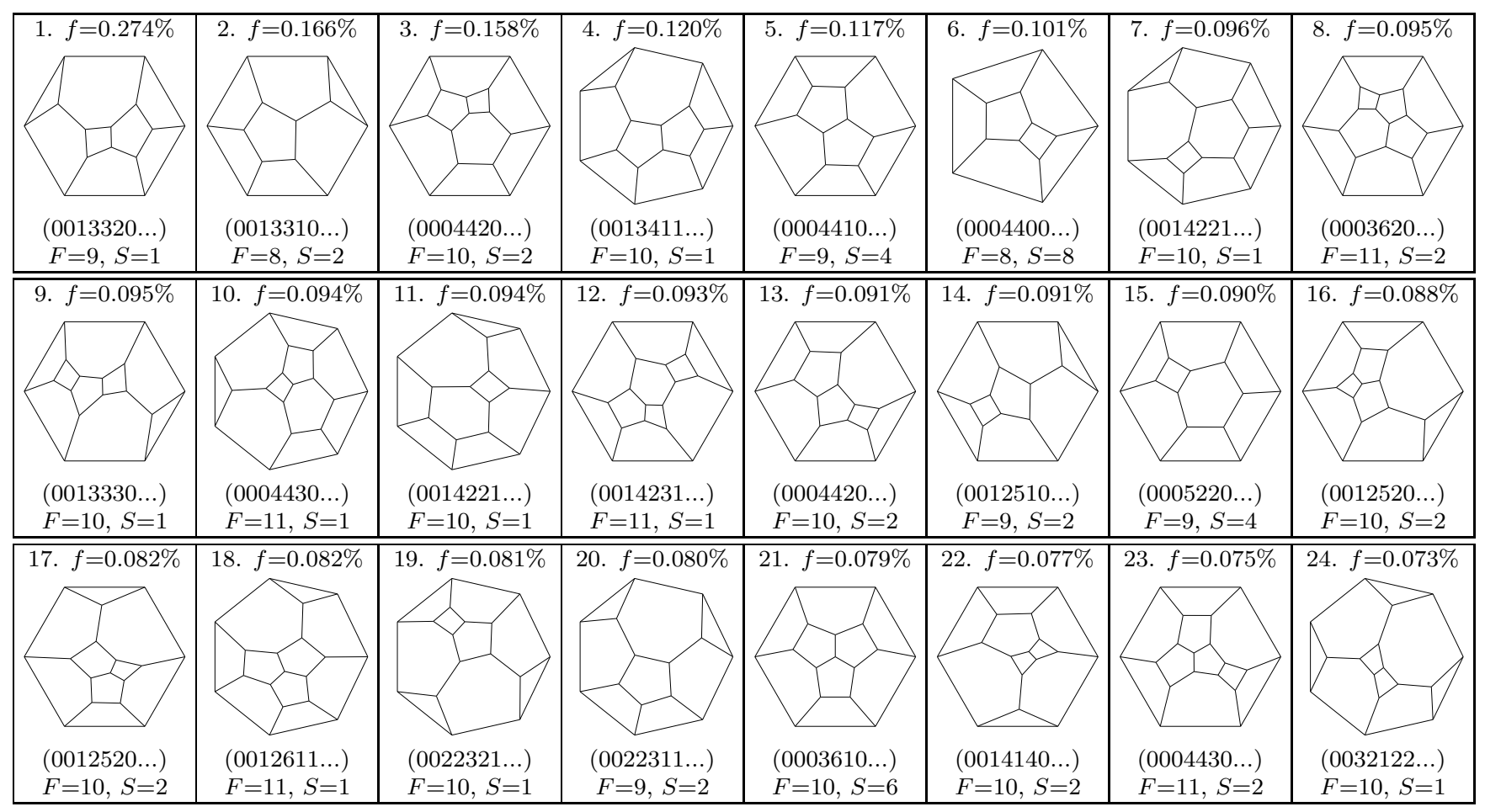

FIG. 6. Schlegel diagrams of the 24 most common topological types among the Poisson-Voronoi cells. Listed for each type is its frequency $f, p$-vector, number of faces $F$, and order $S$ of its associated symmetry group. In these data, there are four pairs of Weinberg vectors which share $p$-vectors.

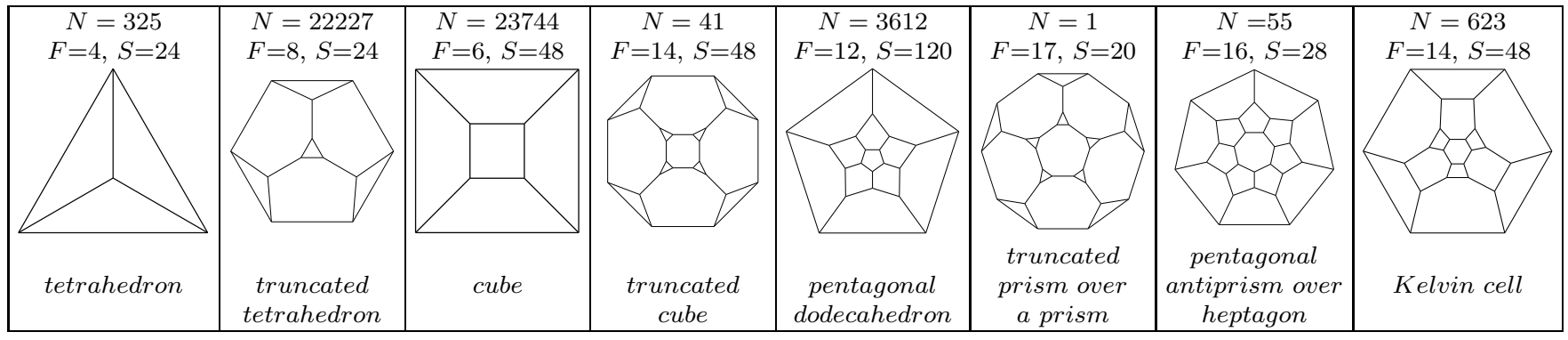

FIG. 7. Highly symmetric polyhedra. For each type, we include the number of times $N$ it appears in the 250,000,000 cell data set, its number of faces $F$, and the order $S$ of its symmetry group.

inconsistency between the diagram and the data, we note that entries 5 and 21 both have only one hexagonal face. Therefore, aside from rotations or reflections, there is no way to redraw identical graphs using a different face as the outside polygon. Entry 6, in contrast, has four pentagonal faces, and the graph can be redrawn with each of those faces as the outside polygon. These contribute additional symmetries which might be initially overlooked when considering the Schlegel diagrams.

\section{G. Cloths and swatches}

The types of topological information considered up to this point concern the configuration of faces and edges on cell surfaces, but not the topology of the network of cells extending throughout the tessellation. This is more difficult to address for at least two reasons. First, much more information is involved in characterizing the topology of the cell network than a single cell. Second, collecting statistics relating to the topological features of the boundary network requires a much larger computational effort.

One approach [34 is to construct and collect statistics of swatches, where a swatch is roughly a collection of labels for the vertices (intersection of four cells) in a portion of the tessellation. The labeling procedure is performed as follows. Let one of the vertices of the structure be designated as the root, and assign a label to this vertex. The swatch is expanded by a canonical procedure that assigns labels to any vertices connected by a single edge to one of the most recently labeled vertices. While performing 


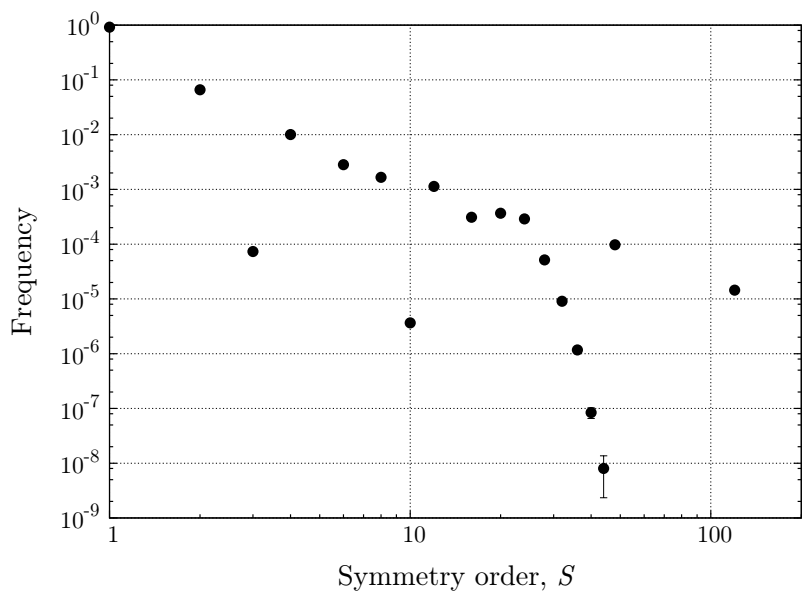

FIG. 8. Distribution of orders of cell symmetry groups. Error bars indicate standard error from the mean; in many cases the error bars are not visible because they are smaller than the points.

this procedure on a quadrivalent Cayley tree would give a single, unique label for every vertex, in practice the network of edges contains loops around every face. The result is that vertices are often assigned multiple labels; the labels of such a vertex are considered to be equivalent, and define an equivalence relation. After $r$ iterations of assigning labels, the set of equivalence relations is known as a swatch of order $2 r$. A swatch contains all of the topological information about the network of cells in the region around the root; as evidence of this, consider that applying the equivalence relations to a labeled quadrivalent Cayley tree exactly reproduces the network of edges. A swatch therefore classifies the topology of the locale, analogous to the way a Weinberg vector classifies a single cell.

For a positive integer $k$, vertices may be randomly selected from the tessellation to serve as root vertices for the construction of swatches of order $k$. The frequencies at which the different types of swatches appear during this sampling gives a probability distribution that effectively describes the distribution of local topological environments. Allowing $k$ to vary over the positive integers gives an infinite set of probability distributions, collectively known as the cloth of the cell network.

The probability distribution for a given value of $k$ further defines a $k$-entropy via the Shannon entropy formula [68]. The $k$-entropy indicates the variability of the local topological environment, and is a well-defined property of an infinite and statistically homogenous 34] cellular structure. The $k$-entropies are reported here as a function of $k$.

We constructed swatches for all $V=1,691,911,665$ vertices in the data set, and report the $k$-entropies for $k=0$ to 8 in Fig. 9. The $k$-entropy of the system is 0 for $k=0,1,2$ since a sufficiently small neighborhood around any vertex is topologically trivial (e.g., an isolated vertex, or a vertex connected to four edges). On the other

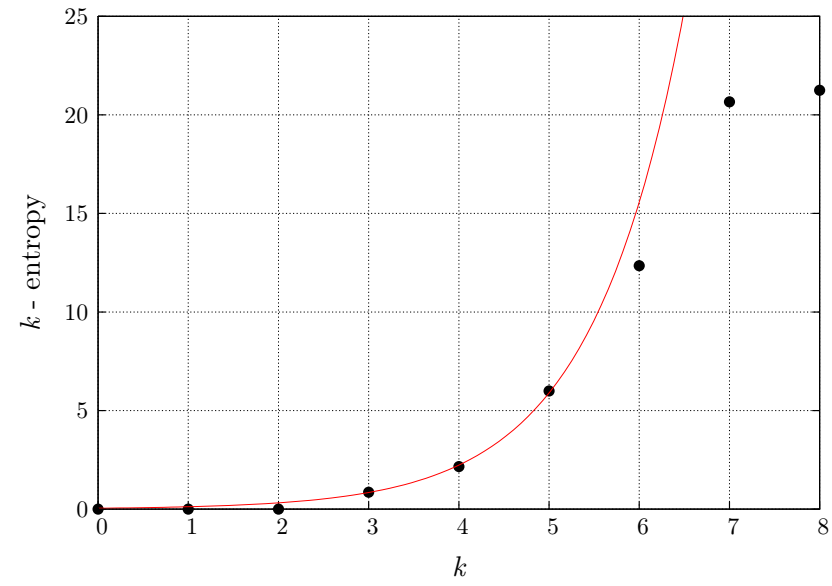

FIG. 9. The $k$-entropy calculated using all $V=1,691,911,665$ vertices in the three-dimensional Poisson-Voronoi structures, containing 250,000,000 cells. The red line is $0.0457 e^{0.972 k}$.

hand, for $k=7,8$ there are so many possible local environments that the number of swatch types is much larger than the number of vertices in the system. As a result, no swatch type is sampled more than once during the sampling procedure, apparently bounding the $k$-entropy from above due to the finite system size. This probably affects the $k$-entropy for $k=6$ as well, where the probability distribution of swatch types appears to be insufficiently sampled. The $k$-entropy for the remaining three values of $k=3,4,5$ is adequately fit by a least-squares procedure to an exponential function.

Suppose that the $k$-entropy is roughly proportional to the natural logarithm of the number of swatch types (this is precisely true in the case of a uniform distribution), and that the exponential form suggested above holds, i.e.,

$$
\ln \left(N_{k}\right) \sim c_{0} \exp \left(c_{1} \cdot k\right)
$$

where $N_{k}$ is the number of swatch types of order $k$. This implies that $N_{k}$ grows roughly as a double exponential,

$$
N_{k} \sim \exp \left(c_{0} \exp \left(c_{1} \cdot k\right)\right) .
$$

Although more data points would certainly help to validate this suggestion, the apparent growth rate means that adequately sampling the $k$-entropy for even $k=6$ is extremely computationally demanding.

Comparing our results with the $k$-entropies for grain growth structures [34], we find that the $k$-entropies of the Poisson-Voronoi tessellation are slightly higher. The slightly higher values are consistent with the greater variability of cell types in the Poisson-Voronoi tessellation, as is evidenced by the differences in the distributions of the $p$-vectors or of the Weinberg vectors for the two structures. That said, the similarity of the $k$-entropies does not imply the similarity of the local topological environments, but only that the amount of variability in the two structures is similar. 


\section{GEOMETRICAL DATA}

One of the most frequently studied geometricaltopological relations is that between the number of faces of a cell and its expected volume. Before considering that relationship, we look at the distribution of volumes over all cells and at the partial distributions of volumes limited to cells with fixed numbers of faces. Likewise, we consider the distribution of surface areas of cells, as well as areas and perimeters of faces.

\section{A. Distribution of volumes}

Despite much interest in understanding the distribution of volumes among three-dimensional PoissonVoronoi cells, few rigorous results are available. Throughout this section we use $x_{v}=v /\langle v\rangle$ to denote normalized cell volumes, where $v$ is the volume of a particular cell and $\langle v\rangle$ is the average volume per cell. Throughout the paper we use $p(x)$ to denote the probability distribution of a variable $x$. Gilbert [17] and Brakke [18] obtained exact integral expressions for the variance of this distribution; numerical integration yields a variance of 0.1790 . Figure [10 shows the distribution of cell volumes in our data set. The distribution exhibits a maximum at roughy $x_{v}=0.831$ with a probability density of $p\left(x_{v}\right)=1.006$. Several suggestions have been made for the form of this distribution.

Hanson [69] suggested that the volume is distributed according to a Maxwell distribution:

$$
p(x)=\frac{32}{\pi^{2}} x^{2} e^{-4 x^{2} / \pi}
$$

Hanson acknowledged the lack of physical motivation to substantiate this suggestion and realized that this form does not provide a particularly good fit to the data for all $x$. In addition, the variance of this distribution, $3 \pi / 8-1$, is not consistent with the exact results of Gilbert [17] and Brakke [18].

Ferenc and Néda 25], motivated by a known result of one-dimensional Poisson-Voronoi structures, and based on the study of 18,000,000 three-dimensional PoissonVoronoi cells, proposed

$$
p(x)=\frac{3125}{24} x^{4} e^{-5 x} .
$$

The variance of this distribution is $1 / 5$ which, again, is inconsistent with the known exact result. Ferenc and Néda acknowledged that this form is empirical and not completely consistent with the true distribution. Because Eqs. (9) and (10) are inconsistent with the exact, known properties of this distribution, we do not consider them further.

Kumar et al. 22] considered a lognormal distribution as an approximation of the Poisson-Voronoi volumes dis- tribution:

$$
p(x)=\frac{1}{x \sqrt{2 \pi} \sigma} \exp \left[-\frac{(\ln x-\mu)^{2}}{2 \sigma^{2}}\right],
$$

where $\mu$ and $\sigma$ are determined by fitting. Using simulation data, Kumar et al. 22] obtained $\sigma=0.4332$ and $\mu=-0.0735$. However, since we know the mean and variance exactly [17, 18, these two parameters are completely determined: $\sigma=0.4058$ and $\mu=-0.0823$. As noted by Kumar et al. [22] and others [70], a lognormal distribution appears to have little physical justification, and given its weakness in fitting the data, can serve only as a rough guide to the actual distribution.

Another suggested form for the Poisson-Voronoi volume distribution is a $\Gamma$ distribution function with one, two, or three fitting parameters. Kiang [71] attempted to extend results known for one-dimensional systems and limited simulation data to suggest a volume distribution of the form:

$$
p(x)=\frac{\gamma}{\Gamma(\gamma)}(\gamma x)^{\gamma-1} e^{-\gamma x}
$$

where $\gamma$ is a constant which Kiang believed to be 6 . Andrade and Fortes [72], using a larger data set, concluded that $\gamma \approx 5.56$. Kumar et al. 22] found $\gamma=5.7869$. All these fits should only be considered approximations, since the variance $\sigma^{2}$ is known exactly. This, then, determines $\gamma=1 / \sigma^{2}=5.586$.

Kumar et al. 22] also suggested a two-parameter version of this distribution,

$$
p(x)=\frac{x^{\gamma-1}}{\beta^{\gamma} \Gamma(\gamma)} e^{-x / \beta} .
$$

Using simulation data, Kumar et al. obtained best fit values of the constants, $\beta=0.1782$ and $\gamma=5.6333$. However, the exact variance results require $\beta=\sigma^{2}=0.1790$ and $\gamma=1 / \sigma^{2}=5.586$. With these values, Eq. (13) reduces to Eq. (12).

Tanemura 24] suggested a three-parameter version of the distribution,

$$
p(x)=\frac{\alpha \beta^{\gamma / \alpha}}{\Gamma(\gamma / \alpha)} x^{\gamma-1} e^{-\beta x^{\alpha}} .
$$

Fitting to simulation data, Tanemura found $\alpha=1.409$, $\beta=2.813$, and $\gamma=4.120$. However, this can be simplified using the exact values for the mean and variance; hence, there is only one free parameter. Fitting to our own data and using these exact results yields $\alpha=1.1580$, which fixes $\beta=4.0681$ and $\gamma=4.7868$.

Figure 10 shows a comparison of the volume distribution for our large data set and the various suggested fits. The parameters in Eqs. (11), (12), and (13) are determined using the known exact results, with the single free parameter in Eq. (14) determined via a least squares fit to our data set.

Inspection of Fig. 10 shows that Eq. (11) does a poor job reproducing the simulation data. Equations 12 and 


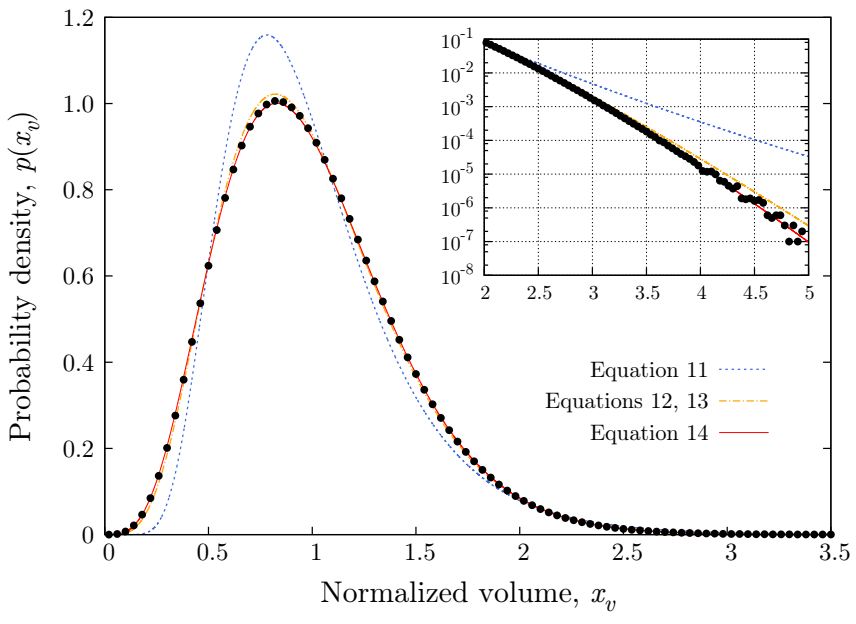

FIG. 10. (Color online) Distribution of normalized cell volumes, $x_{v}=v /\langle v\rangle$. The standard deviation of the data set is 0.4231 , consistent with analytical results to within numerical accuracy. The curves represent suggested forms of the distribution, as described in the text. The inset shows a subset of the data on a semilogarithmic plot.

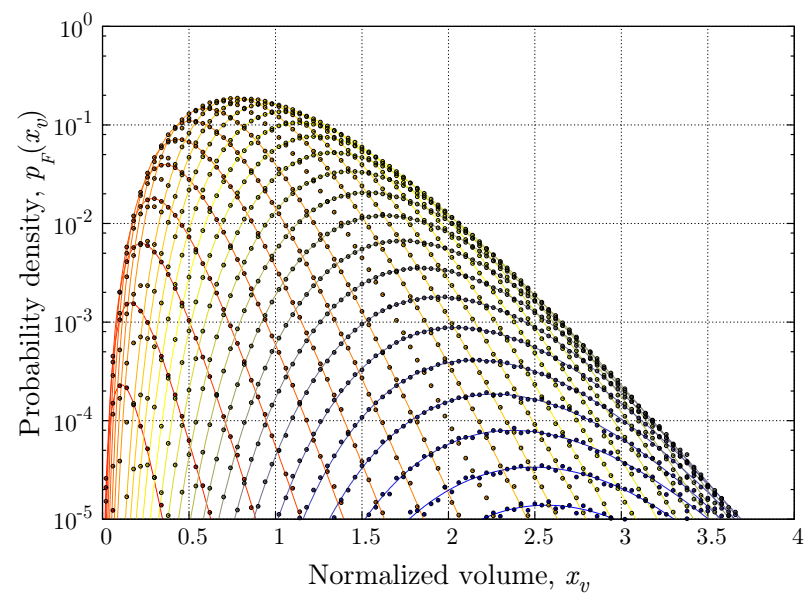

FIG. 11. (Color online) Partial distributions $p_{F}\left(x_{v}\right)$ of cell volumes for each number of faces $F$. The red-most curve, at the bottom left of the plot, corresponds to $F=5$; the bluemost curve, at the bottom right of the plot, corresponds to $F=32$. Data were binned in intervals of width 0.04 .

13 exhibit systematic errors compared with the simulation data (see both the peak position and the large $x$ behavior), although they are far superior to Eq. (11). The adjustable three-parameter $\Gamma$ distribution function [Eq. 14)] provide a best fit to the data.

We next consider the partial distributions $p_{F}\left(x_{v}\right)$ of cell volumes for each number of faces $F$; the partial distributions are normalized so $p(x)=\sum_{F=1}^{\infty} p_{F}\left(x_{v}\right)$. Data for $4 \leq F \leq 32$ are shown in Fig. [11, a semi-log scale is used to help differentiate the data for very small and very large volumes. Tanemura [24] used a relatively large

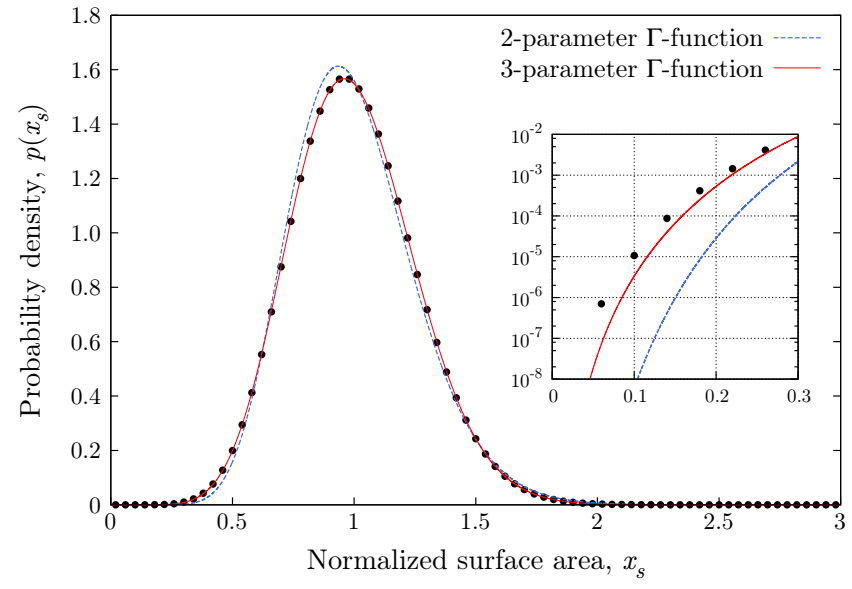

FIG. 12. (Color online) Distribution of surface areas among all cells. Data were binned in intervals of width 0.04 . The standard deviation is 0.254 , shown to three decimal places. The inset shows a subset of the data on a semilogarithmic plot.

data set ( 5 million cells) and suggested that each of these partial distributions could be accurately described by the three-parameter $\Gamma$ function considered earlier [Eq. (14)], where $\alpha, \beta$, and $\gamma$ for each curve are parameters that depend on $F$. We test this suggestion using a least squares fit to obtain parameters $\alpha, \beta$, and $\gamma$ for each $F$. Figure 11 shows least squares fits of Eq. (14) for each $F$. Obtained parameters are provided in Table IX of the Supplemental Material. While we know of no theoretical reason to expect this form, it appears to match the data very well.

\section{B. Distribution of surface areas}

We next consider the distribution of surface areas over all cells. In this section we use $x_{s}=s /\langle s\rangle$ to denote normalized surface area, where $s$ is the surface area of a particular cell and $\langle s\rangle$ is the average surface area per cell. Brakke [18] provided an integral equation for the variance of this distribution, and numerically evaluated it to be 0.064679 . Our data reproduce this exact result to within $0.0001 \%$. Figure 12 plots the distribution of surface areas in our data set. The curve appears to peak at roughly $x_{s}=0.96$ with a probability density of $p\left(x_{s}\right)=1.57$.

Kumar et al. suggested that this distribution can be described by a two-parameter $\Gamma$ function [Eq. (13)] with fitted parameters $\alpha=15.4847$ and $\beta=0.06490$. However, since both the mean and variance are known, there are no degrees of freedom in fitting two parameters. The analytic constraints yield $\alpha=15.461$ and $\beta=0.06468$.

If we consider a three-parameter $\Gamma$ function [Eq. (14)], then we are left with one degree of freedom in choosing the parameters. A least squares fit finds that $\alpha=1.845$, $\beta=4.416$, and $\gamma=8.557$ fit the data most closely, while satisfying the known analytic constraints. Figure 


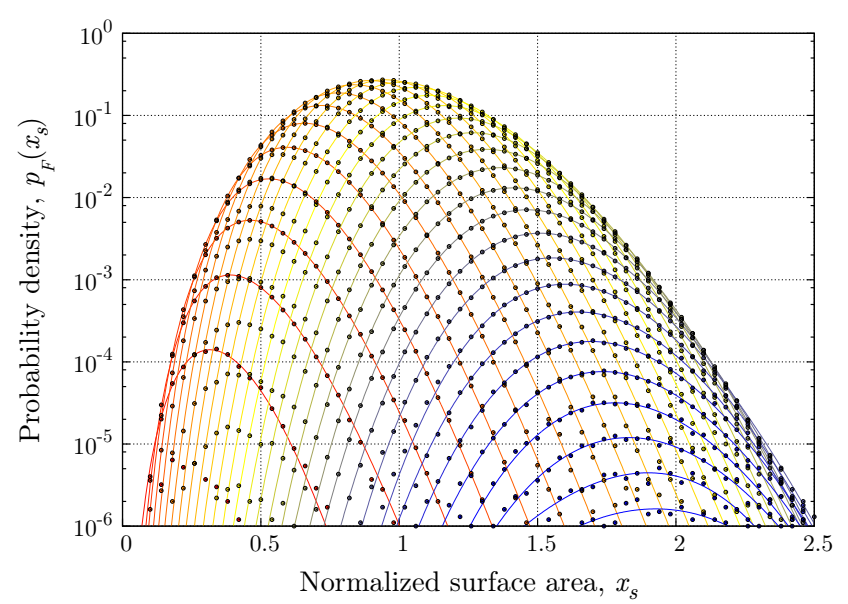

FIG. 13. (Color online) Partial distributions $p_{F}\left(x_{s}\right)$ of cell surface areas for each number of faces $F$. The red-most curve, at the bottom left of the plot, corresponds to $F=4$; the bluemost curve, at the bottom right of the plot, corresponds to $F=33$. Data were binned in intervals of width 0.04 .

12 shows both fits and the collected data. Although the three-parameter version slightly underestimates $p(x)$ for small $x$, as can be seen on the inset plot, overall it provides excellent agreement with the data.

Figure 13 shows the partial distributions $p_{F}\left(x_{s}\right)$ of surface areas for cells with fixed numbers of faces. We show the data on a semi-log plot to focus attention on data of very large and small surface areas. Tanemura [24] suggested that these distributions could also be accurately described by the three-parameter $\Gamma$ function considered earlier [Eq. (14)], where $\alpha, \beta$, and $\gamma$ for each curve are parameters that depend on $F$. We test this suggestion using a least squares fit to obtain parameters $\alpha, \beta$, and $\gamma$ for each $F$. Figure 11 shows least squares fits of Eq. (14) for each $F$; the parameters are provided in Table $\mathrm{X}$ of the Supplemental Material. While we cannot provide justification to expect this form, it appears to match the data very well.

\section{Distribution of face areas}

We next consider the distribution of areas of faces. In this section we use $x_{a}=a /\langle a\rangle$ to denote normalized areas, where $a$ is the area of a particular face and $\langle a\rangle$ is the average area over all faces. Brakke [18] provided an integral expression for the variance of this distribution; numerical evaluation shows that it is equal to 1.01426 . Our data reproduce this exact result to within $0.005 \%$. The black curves in Fig. 14 show the distribution of areas among all faces in our data set. Unlike the distributions considered earlier, this one is far from symmetric; instead, it is strongly biased towards faces with very small areas.

We also consider the partial distributions $p_{n}\left(x_{a}\right)$ of

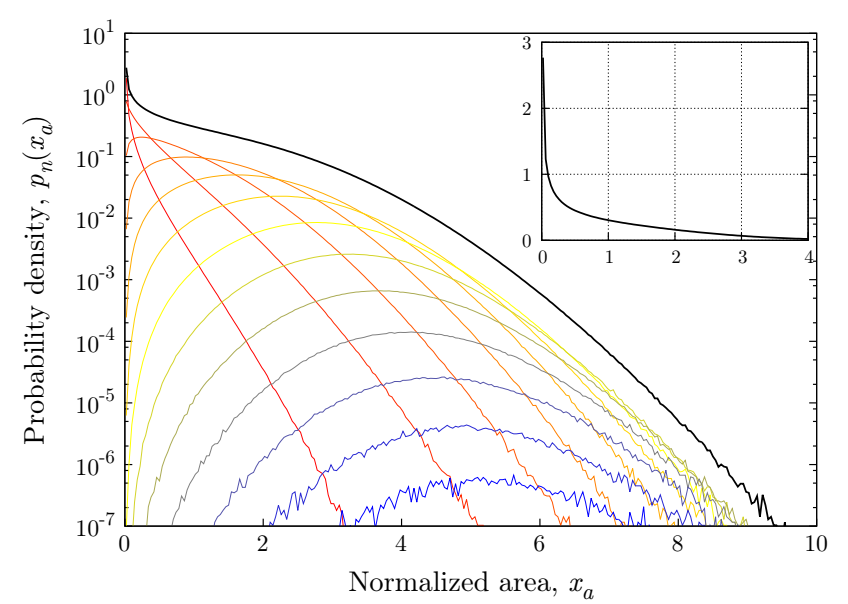

FIG. 14. (Color online) Partial distributions $p_{n}\left(x_{a}\right)$ of face areas for each number of edges $n$. The red-most curve, located to the left of the other curves, corresponds to $n=3$; the blue-most curve, located at the bottom-center of the plot, corresponds to $n=15$. The black curves show the distribution of areas summed over all $n$. Data were binned in intervals of width 0.04 .

areas limited to faces with fixed numbers of edges $n$. The colored curves in Fig. 14 show these distributions. It appears from the figure that $p_{n}(0)>0$ for $n=3$ and 4 . Hence, these curves cannot be fitted using a $\Gamma$ function [Eq. (14)], for which $p(0)$ always evaluates to 0 .

\section{Distribution of face perimeters}

Last, we consider the distribution of perimeters of faces. In this section we use $x_{l}=l /\langle l\rangle$ to denote a normalized perimeter, where $l$ is the perimeter of a particular face and $\langle l\rangle$ is the average perimeter over all faces. Again, Brakke [18] derived an exact analytical expression for the variance that evaluates to 0.2898 . Our data reproduce this result to within $0.004 \%$. The black curves in Fig. 15 show the distribution of perimeters among all faces in our data set. The shape of this figure is similar to that calculated analytically by Brakke [73] for the distribution of edge lengths in two-dimensional PoissonVoronoi structures.

We also consider the partial distributions $p_{n}\left(x_{l}\right)$ of perimeters limited to faces with fixed numbers of edges $n$. The colored curves in Fig. 15 show these distributions. It appears from the data that $p_{n}(0)>0$ for $n=3$; this implies that the partial perimeter distributions cannot be fitted to a $\Gamma$ function. 


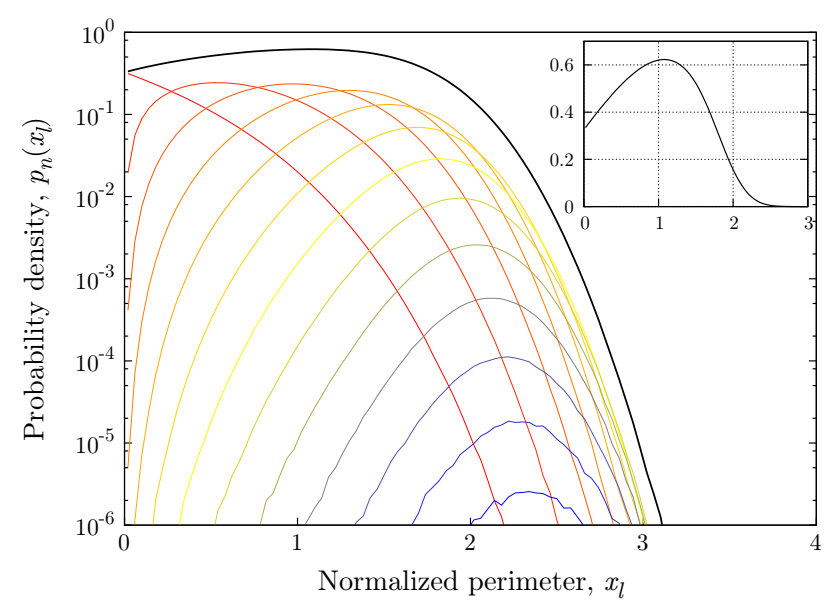

FIG. 15. (Color online) Partial distributions $p_{n}\left(x_{l}\right)$ of face perimeters for each number of edges $n$. The red-most curve, located to the left of the other curves, corresponds to $n=3$; the blue-most curve, located at the bottom-center of the plot, corresponds to $n=15$. The black curves show the distribution of perimeters summed over all $n$. Data were binned in intervals of width 0.04 .

\section{CORRELATIONS BETWEEN GEOMETRY AND TOPOLOGY}

We now consider how the average volume and surface area of a cell depend on its number of faces $F$, and how the average area and perimeter of a face depend on its number of edges $n$. In two-dimensional systems, the study of this type of relationship was pioneered by Lewis [74], who observed in some natural structures that the area of a cell was proportional to its number of edges.

Figure 16 shows the average volume of a cell as a function of its number of faces; we use $\left\langle x_{v}\right\rangle_{F}$ to denote the average volume of cells with $F$ faces. Based on a data set with 102,000 cells, Kumar et al. 22 suggested that $\left\langle x_{v}\right\rangle_{F}=A F^{b}$, where $A$ and $b$ are fitting parameters. Kumar et al. found $A=0.0164$ and $b=1.498$; the more extensive data collected here yield similar values, $A=0.0176$ and $b=1.468$. The curve appears to fit the data well for $10 \leq F \leq 20$, though not for large or small $F$.

A similar relation might be considered for the average surface area of a cell. Based on simulation results, Kumar et al. 22] suggested that $\left\langle x_{s}\right\rangle_{F}=A F^{b}$, where $\left\langle x_{s}\right\rangle_{F}$ is the average surface area of cells with $F$ faces and $A$ and $b$ are fitting parameters (Fig. 17). Kumar et al. found $A=$ 0.09645 and $b=0.8526$; our data yield similar values, $A=0.0993$ and $b=0.843$. This curve too appears to fit the data well for $10 \leq F \leq 20$, though also fails for large and small $F$.

Finally we turn to the dependance of the expected area and perimeter of a face on its number of edges $n$, illustrated in Figs. 18 and 19. Although the exact forms of these relationships cannot be determined, it is clear that

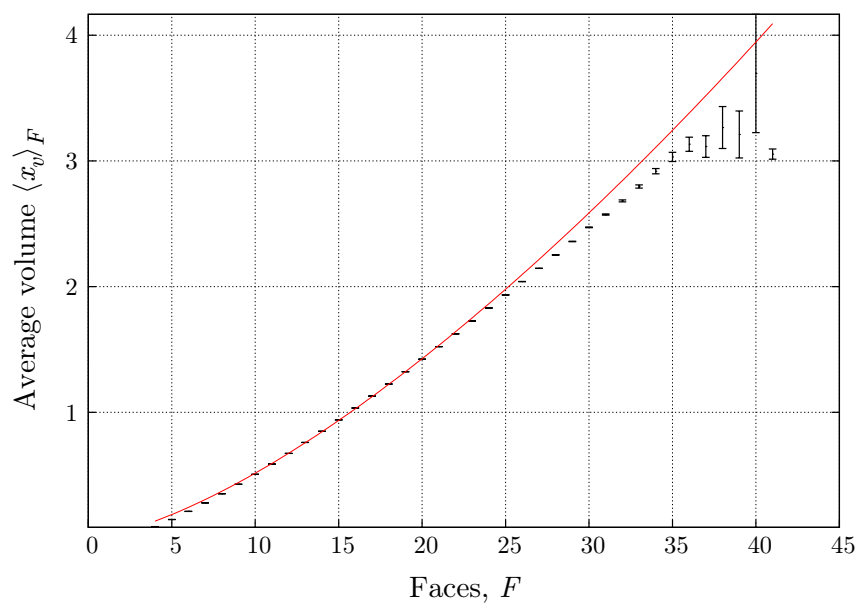

FIG. 16. Average normalized volume $\left\langle x_{v}\right\rangle_{F}$ as a function of number of faces $F$; error bars indicate standard error from the mean. The red curve is a least squares fit to $A F^{b}$, as explained in the text.

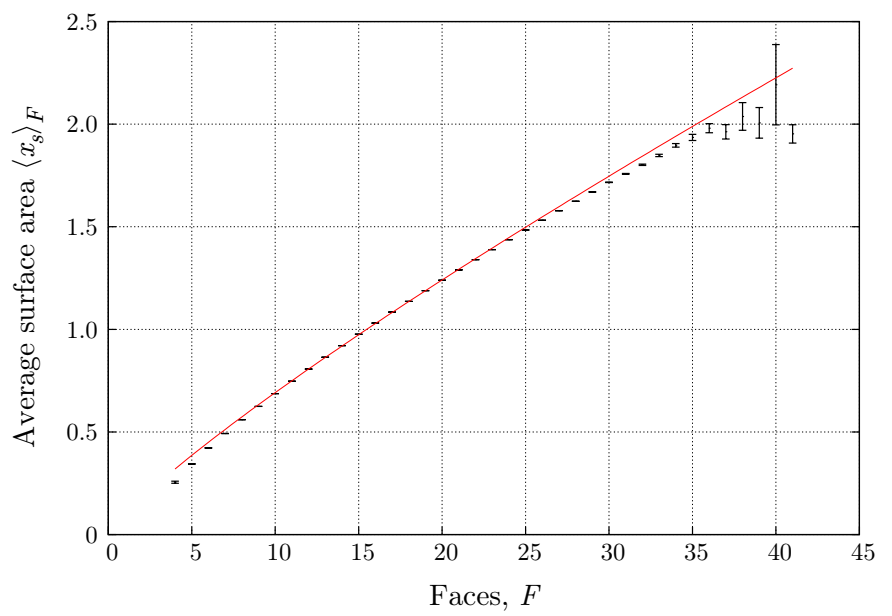

FIG. 17. Average normalized surface area $\left\langle x_{s}\right\rangle_{F}$ as a function of number of faces $F$; error bars indicate standard error from the mean. The red curve is a least squares fit to $A F^{b}$, as explained in the text.

neither the average area nor perimeter of a face increase linearly with $n$.

\section{CONCLUSIONS}

Poisson-Voronoi networks are widely used across the physical and biological sciences as canonical cell structures. While two-dimensional Poisson-Voronoi networks have been widely studied and often used as surrogates for three-dimensional applications, such three-dimensional networks have been much less widely examined. In this report, we have provided a much more complete charac- 


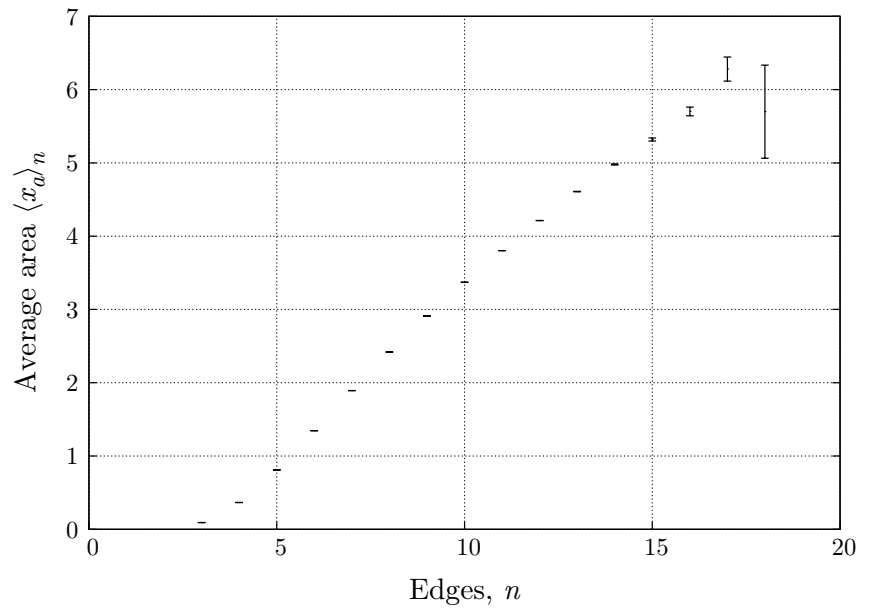

FIG. 18. Average normalized area $\left\langle x_{a}\right\rangle_{n}$ as a function of number of edges $n$; error bars indicate standard error from the mean.

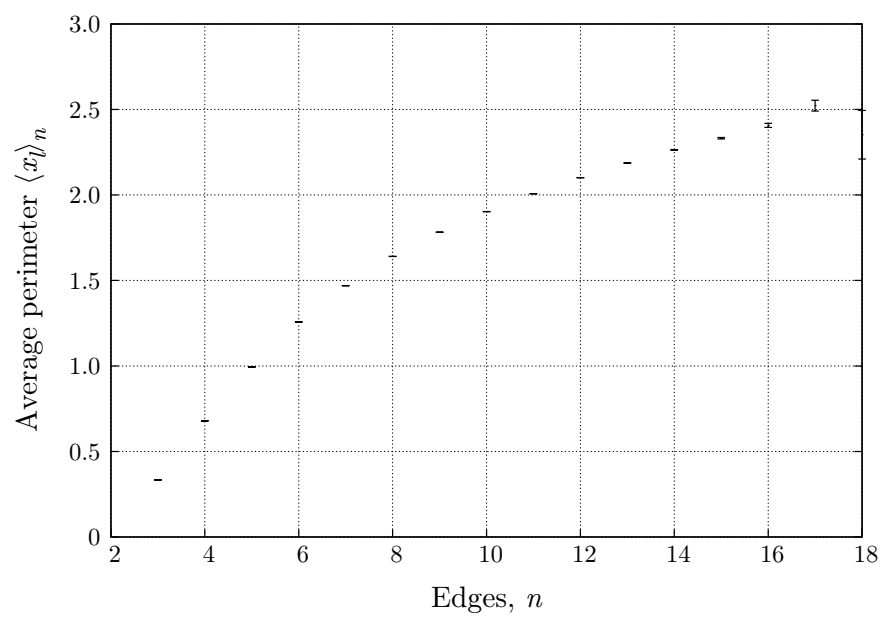

FIG. 19. Average normalized perimeter $\left\langle x_{l}\right\rangle_{n}$ as a function of number of edges $n$; error bars indicate standard error from the mean.

terization of three-dimensional Poisson-Voronoi networks than exists in the literature.

In particular, we report a wide range of statistical properties of three-dimensional Poisson-Voronoi structures containing a combined total of 250,000,000 cells. The data demonstrate that although the Poisson-Voronoi structure is generated using a random distribution of points, it exhibits a rich topological and geometrical structure.

The size of the data set considered here has enabled us to resolve properties of such structures that have been impossible to investigate previously. While some of the results corroborate earlier work at much higher precision, the results also clearly contradict other conjectures.

In particular, we found that the natural extension of the Aboav-Weaire relation to three dimensions is not consistent with our very large data set, contrary to what was previously reported [22, 50]. In particular, for $F<$ 12 faces, the average number of faces of a cell's neighbors increases with the number of faces $F$ of a central cell. This is consistent with recent theoretical results [51, 52].

Considering more refined topological data, we observed that some $p$-vectors appear significantly more frequently than others. We also observed that even when considering a fixed $p$-vector, not all topological types appear with equal frequencies. Understanding such topological distributions may provide new insight into the topological structure of other natural cellular structures and the forces under which those systems evolve. One particularly interesting set of results shows that the order of the symmetry groups of the three-dimensional PoissonVoronoi cells shows clear trends that can be used to distinguish it from other types of cellular networks.

Our data set supports the conjecture of Tanemura 24] regarding the distribution of cell volumes and surface areas when restricted to cells with fixed numbers of faces. In particular, a three-parameter $\Gamma$ function [Eq. (14)] appears to fit these data precisely. This equation also appears to fit the distribution of volumes over all cells. However, this functional form does not accurately describe the distribution of cell surface areas or cell face areas and perimeters.

We considered the dependence of the expected volume and surface area of a cell on its number of faces. The data presented here counters conjectures of Kumar et al. 22. regarding the form of this relationship. Unfortunately, we were unable to provide a well-founded alternative.

Extensive geometrical and topological statistics from our data structures are included in the Supplemental Material and an extensive set of measures of the cells in the entire 250,000,000 cell data set is available online at [75].

Acknowledgments. We thank Ken Brakke for providing computer programs to generate Poisson-Voronoi structures, and for ongoing support of his Surface Evolver program. Most of the computations reported herein were performed using the computational resources of the Institute for Advanced Study.
[1] A. Okabe, B. N. Boots, K. Sugihara, and S. N. Chiu, Spatial Tessellations: Concepts and Applications of Voronoi Diagrams (Wiley \& Sons, Chichester, UK, 1992).

[2] D. Stoyan, W. S. Kendall, J. Mecke, and L. Ruschendorf, Stochastic Geometry and Its Applications (Wiley
Chichester, 1995).

[3] V. Icke, in Large-Scale Structures in the Universe Observational and Analytical Methods (Springer, Berlin, 1988) pp. 257-266.

[4] S. Yoshioka and S. Ikeuchi, The Astrophysical Journal 
341, 16 (1989).

[5] M. Ramella, W. Boschin, D. Fadda, and M. Nonino, arXiv preprint astro-ph/0101411 (2001).

[6] M. Tanemura and M. Hasegawa, Journal of Theoretical Biology 82, 477 (1980).

[7] R. A. Fischer and R. E. Miles, Mathematical Biosciences 18, 335 (1973).

[8] A. L. Mackay, Journal of Microscopy 95, 217 (2011).

[9] J. Finney, Proceedings of the Royal Society of London A 319, 479 (1970).

[10] H. G. E. Hentschel, V. Ilyin, N. Makedonska, I. Procaccia, and N. Schupper, Phys. Rev. E 75, 050404 (2007).

[11] V. A. Luchnikov, N. N. Medvedev, Y. I. Naberukhin, and H. R. Schober, Phys. Rev. B 62, 3181 (2000).

[12] D. J. Daley and D. Vere-Jones, An Introduction to the Theory of Point Processes: Volume I: Elementary Theory and Methods, Vol. 1 (Springer, Berlin, 2003).

[13] D. J. Daley and D. Vere-Jones, An Introduction to the Theory of Point Processes: Volume II: General Theory and Structure (Springer, Berlin, 2007).

[14] J. F. C. Kingman, Poisson Processes (Oxford University Press, Oxford, 1992).

[15] D. D. R. Cox and V. Isham, Point Processes, Vol. 12 (CRC Press, Boca Raton, FL, 1980).

[16] J. L. Meijering, Philips Research Reports 8, 270 (1953).

[17] E. N. Gilbert, Annals of Mathematical Statistics 33, 958 (1962).

[18] K. A. Brakke, "Statistics of Three Dimensional Random Voronoi Tessellations," (1987).

[19] J. Møller, Lectures on Random Voronoi Tessellations (Springer, Berlin, 1994).

[20] J. Møller and D. Stoyan, Stochastic Geometry and Radom Tessellations, Tech. Rep. (Department of Mathematical Sciences, Aalborg University, 2007).

[21] P. Calka, in New Perspectives in Stochastic Geometry, edited by W. S. Kendall and I. Molchanov (Oxford University Press, Oxford, 2010).

[22] S. Kumar, S. K. Kurtz, J. R. Banavar, and M. G. Sharma, Journal of Statistical Physics 67, 523 (1992).

[23] K. Marthinsen, Materials Characterization 36, 53 (1996).

[24] M. Tanemura, Forma 18, 221 (2003).

[25] J. S. Ferenc and Z. Néda, Physica A: Statistical Mechanics and its Applications 385, 518 (2007).

[26] A. Thorvaldsen, in Materials Science Forum, Vol. 94 (Trans Tech, Dürnten, 1992) pp. 307-312.

[27] N. Reis, A. C. Ferro, and J. C. Pereira, in Materials Science Forum, Vol. 514 (Trans Tech Publ, 2006) pp. 1488-1492.

[28] G. E. Schröder-Turk, W. Mickel, S. C. Kapfer, F. M. Schaller, B. Breidenbach, D. Hug, and K. Mecke, arXiv preprint 1009.2340 (2010).

[29] S. C. Kapfer, W. Mickel, F. M. Schaller, M. Spanner, C. Goll, T. Nogawa, N. Ito, K. Mecke, and G. E. Schröder-Turk, Journal of Statistical Mechanics: Theory and Experiment 2010, P11010 (2010).

[30] G. E. Schröder-Turk, W. Mickel, M. Schröter, G. W. Delaney, M. Saadatfar, T. J. Senden, K. Mecke, and T. Aste, EPL (Europhysics Letters) 90, 34001 (2010).

[31] See Supplemental Material at the end of this article for extensive geometrical and topological statistics from our data structures, including those used in the figures.

[32] K. Brakke, Personal communication (2007).

[33] E. A. Lazar, J. K. Mason, R. D. MacPherson, and D. J.
Srolovitz, Phys. Rev. Lett. 109, 095505 (2012)

[34] J. K. Mason, E. A. Lazar, R. D. MacPherson, and D. J. Srolovitz, Phys. Rev. E 86, 051128 (2012).

[35] C. S. Smith, in Metal Interfaces (American Society for Metals, Cleveland, OH, 1952) pp. 108-110.

[36] F. N. Rhines, K. R. Craig, and R. T. DeHoff, Metallurgical Transactions 5, 413 (1974).

[37] D. Aboav, Metallography 3, 383 (1970).

[38] D. Weaire, Metallography 7, 157 (1974).

[39] B. Jeune and D. Barabé, Annals of Botany 82, 577 (1998).

[40] J. C. M. Mombach, R. M. C. de Almeida, and J. R. Iglesias, Phys. Rev. E 48, 598 (1993).

[41] D. L. Weaire and S. Hutzler, The Physics of Foams (Oxford University Press, 1999).

[42] S. J. Mejía-Rosales, R. Gámez-Corrales, B. I. Ivlev, and J. Ruiz-García, Physica A: Statistical Mechanics and its Applications 276, 30 (2000).

[43] F. Elias, C. Flament, J.-C. Bacri, O. Cardoso, and F. Graner, Phys. Rev. E 56, 3310 (1997).

[44] J. Earnshaw and D. Robinson, Phys. Rev. Lett. 72, 3682 (1994).

[45] P. Moriarty, M. D. R. Taylor, and M. Brust, Phys. Rev. Lett. 89, 248303 (2002).

[46] B. Boots, Metallography 15, 53 (1982).

[47] S. Kumar and S. K. Kurtz, Materials Characterization 31, 55 (1993).

[48] H.-J. Hilhorst, arXiv preprint cond-mat/0509409 (2005).

[49] H.-J. Hilhorst, Journal of Physics A 39, 7227 (2006).

[50] M. Fortes, Philosophical Magazine Letters 68, 69 (1993).

[51] H. Hilhorst, Journal of Statistical Mechanics: Theory and Experiment 2009, P08003 (2009).

[52] J. K. Mason, R. Ehrenborg, and E. A. Lazar, Journal of Physics A 45, 065001 (2012).

[53] D. Barnette, Journal of Combinatorial Theory 7, 99 (1969).

[54] E. B. Matzke, American Journal of Botany 33, 58 (1946).

[55] W. M. Williams and C. S. Smith, Transactions of the Metallurgical Society of AIME 194, 755 (1952).

[56] A. M. Kraynik, D. A. Reinelt, and F. van Swol, Phys. Rev. E 67, 031403 (2003).

[57] L. Ratke and P. W. Voorhees, Growth and Coarsening: Ostwald Ripening in Material Processing (Springer, Berlin, 2002).

[58] L. Weinberg, in Proceedings: Third Annual Allerton Conference on Circuit and System Theory (University of Illinois, Monticello, Illinois, 1965) pp. 733-744.

[59] L. Weinberg, IEEE Trans. Circuit Theory 13, 142 (1966).

[60] L. Weinberg, SIAM Journal on Applied Mathematics 14, 729 (1966).

[61] V. Schlegel, Theorie der homogen zusammengesetzten Raumgebilde, Vol. 44 (Druck von E. Blochmann \& Sohn, Dresden, 1883).

[62] V. Schlegel, in Catalog Mathematischer Modelle für den Höheren, edited by M. Schilling (Halle a/S, Germany, 1903) 6th ed.

[63] F. Lutz, "The Manifold Page," http://page.math. tu-berlin.de/ lutz/stellar/ (2013).

[64] W. Thomson, Acta Mathematica 11, 121 (1887).

[65] W. Thomson, Proceedings of the Royal Society of London 55, 1 (1894).

[66] T. Hales, Discrete \& Computational Geometry 25, 1 (2001).

[67] E. Lazar, J. Mason, R. MacPherson, and D. Srolovitz, 
Acta Materialia 59, 6837 (2011).

[68] C. E. Shannon, Bell System Technical Journal 27, 379 (1948).

[69] H. G. Hanson, Journal of Statistical Physics 30, 591 (1983).

[70] M. Fátima Vaz and M. Fortes, Scripta Metallurgica 22, 35 (1988).

[71] T. Kiang, Zeitschrift fur Astrophysik 64, 433 (1966).
[72] P. Andrade and M. Fortes, Philosophical Magazine B 58, 671 (1988).

[73] K. A. Brakke, "Statistics of Random Plane Voronoi Tessellations," (1987).

[74] F. Lewis, Anatomical Record 38, 341 (1928).

[75] E. A. Lazar, "Statistics of Three-Dimensional PoissonVoronoi Structures," http://web.math.princeton.edu/ lazar/voronoi.html (2013). 


\section{Supplemental Material}

In the following pages, we report a wide range of data describing the topology of faces, cells, and cell edge networks of three-dimensional Poisson-Voronoi structures. We include the distribution of face edges, cell faces, $p$-vectors, topological types, and symmetry orders. We also report the distribution of cell areas and perimeters, and of cell volumes and surface areas. Then, we break these distributions down further into distributions for faces with a fixed number of sides, and to cells with a fixed number of faces. The complete data are available online at http://web.math.princeton.edu/ lazar/voronoi.html.

\begin{tabular}{|l|l|}
\hline File & Description \\
\hline \hline all_pvectors.data & Distribution of all 375,410 observed $p$-vectors. \\
\hline top_10000_wvectors.data & Distribution of 10,000 most common Weinberg vectors. \\
\hline edges_dist_areas_perims.data & Distribution of edges, and average area and perimeter. \\
\hline faces_dist_vols_areas.data & Distribution of faces, and average volume and surface area. \\
\hline p_vectors_and_types.data & Counts of possible and observed $p$-vectors and topological types. \\
\hline symmetries.data & Distribution of symmetry orders. \\
\hline areas_distribution.data & Distribution of areas of all faces. \\
\hline faces_areas_distributions.data & Distribution of areas of all faces with fixed number of edges. \\
\hline perims_distribution.data & Distribution of perimeters of all faces. \\
\hline faces_perim_distributions.data & Distribution of perimeters of all faces with fixed number of edges. \\
\hline volumes_distribution.data & Distribution of volumes of all cells. \\
\hline faces_volumes_distributions.data & Distribution of volumes of all cells with fixed number of faces. \\
\hline surface_areas_distribution.data & Distribution of surface areas of all cells. \\
\hline faces_surface_areas_distributions.data & Distribution of surface areas of all cells with fixed number of faces. \\
\hline
\end{tabular}




\begin{tabular}{|c|c|c|c|c|}
\hline & $p$-vector & $F$ & $N$ & $\bar{f}$ \\
\hline 1 & $(001343100 \ldots)$ & 12 & 970356 & 0.00388 \\
\hline 2 & $(001342100 \ldots)$ & 11 & 854284 & 0.00342 \\
\hline 3 & $(001433200 \ldots)$ & 13 & 744186 & 0.00298 \\
\hline 4 & $(001344100 \ldots)$ & 13 & 722099 & 0.00289 \\
\hline 5 & $(001423100 \ldots)$ & 11 & 718819 & 0.00288 \\
\hline 6 & $(002333110 \ldots)$ & 13 & 710824 & 0.00284 \\
\hline 7 & $(001332000 \ldots)$ & 9 & 686096 & 0.00274 \\
\hline 8 & $(000442000 \ldots)$ & 10 & 662391 & 0.00265 \\
\hline 9 & $(001352200 \ldots)$ & 13 & 657484 & 0.00263 \\
\hline 10 & $(002233100 \ldots)$ & 11 & 652330 & 0.00261 \\
\hline 11 & $(001432200 \ldots)$ & 12 & 645966 & 0.00258 \\
\hline 12 & $(001353200 \ldots)$ & 14 & 644724 & 0.00258 \\
\hline 13 & $(002332110 \ldots)$ & 12 & 640657 & 0.00256 \\
\hline 14 & $(001422100 \ldots)$ & 10 & 633915 & 0.00254 \\
\hline 15 & $(002322200 \ldots)$ & 11 & 629166 & 0.00252 \\
\hline 16 & $(002242200 \ldots)$ & 12 & 619653 & 0.00248 \\
\hline 17 & $(002342210 \ldots)$ & 14 & 617595 & 0.00247 \\
\hline 18 & $(001443110 \ldots)$ & 14 & 608920 & 0.00244 \\
\hline 19 & $(000443000 \ldots)$ & 11 & 597989 & 0.00239 \\
\hline 20 & $(002343210 \ldots)$ & 15 & 582316 & 0.00233 \\
\hline 21 & $(001442110 \ldots)$ & 13 & 579534 & 0.00232 \\
\hline 22 & $(001424100 \ldots)$ & 12 & 578374 & 0.00231 \\
\hline 23 & $(001434200 \ldots)$ & 14 & 557186 & 0.00223 \\
\hline 24 & $(002243200 \ldots)$ & 13 & 543534 & 0.00217 \\
\hline 25 & $(002323200 \ldots)$ & 12 & 531980 & 0.00213 \\
\hline 26 & $(002232100 \ldots)$ & 10 & 524974 & 0.00210 \\
\hline 27 & $(002423210 \ldots)$ & 14 & 508054 & 0.00203 \\
\hline 28 & $(002334110 \ldots)$ & 14 & 504469 & 0.00202 \\
\hline 29 & $(001252000 \ldots)$ & 10 & 502450 & 0.00201 \\
\hline 30 & $(000533100 \ldots)$ & 12 & 496934 & 0.00199 \\
\hline 31 & $(001263100 \ldots)$ & 13 & 494245 & 0.00198 \\
\hline 32 & $(001341100 \ldots)$ & 10 & 488878 & 0.00196 \\
\hline 33 & $(002234100 \ldots)$ & 12 & 482640 & 0.00193 \\
\hline 34 & $(001354200 \ldots)$ & 15 & 479810 & 0.00192 \\
\hline 35 & $(001345100 \ldots)$ & 14 & 477182 & 0.00191 \\
\hline 36 & $(001334000 \ldots)$ & 11 & 475735 & 0.00190 \\
\hline 37 & $(002422210 \ldots)$ & 13 & 473982 & 0.00190 \\
\hline 38 & $(002333300 \ldots)$ & 14 & 471010 & 0.00188 \\
\hline 39 & $(002324200 \ldots)$ & 13 & 468805 & 0.00188 \\
\hline 40 & $(001442300 \ldots)$ & 14 & 463908 & 0.00186 \\
\hline 41 & $(001444110 \ldots)$ & 15 & 462925 & 0.00185 \\
\hline 42 & $(001443300 \ldots)$ & 15 & 461700 & 0.00185 \\
\hline 43 & $(002332300 \ldots)$ & 13 & 449667 & 0.00180 \\
\hline 44 & $(001351200 \ldots)$ & 12 & 448514 & 0.00179 \\
\hline 45 & $(000453100 \ldots)$ & 13 & 445787 & 0.00178 \\
\hline 46 & $(001533210 \ldots)$ & 15 & 437472 & 0.00175 \\
\hline 47 & $(001333000 \ldots)$ & 10 & 433409 & 0.00173 \\
\hline 48 & $(001453210 \ldots)$ & 16 & 429016 & 0.00172 \\
\hline 49 & $(002344210 \ldots)$ & 16 & 424623 & 0.00170 \\
\hline 50 & $(001452210 \ldots)$ & 15 & 417387 & 0.00167 \\
\hline 51 & $(001331000 \ldots)$ & 8 & 414904 & 0.00166 \\
\hline 52 & $(002244200 \ldots)$ & 14 & 413495 & 0.00165 \\
\hline 53 & $(001262100 \ldots)$ & 12 & 413322 & 0.00165 \\
\hline 54 & $(000454100 \ldots)$ & 14 & 407166 & 0.00163 \\
\hline 55 & $(000452100 \ldots)$ & 12 & 400062 & 0.00160 \\
\hline 56 & $(003232210 \ldots)$ & 13 & 389187 & 0.00156 \\
\hline 57 & $(002432310 \ldots)$ & 15 & 383600 & 0.00153 \\
\hline 58 & $(003233210 \ldots)$ & 14 & 383450 & 0.00153 \\
\hline 59 & $(000534100 \ldots)$ & 13 & 382470 & 0.00153 \\
\hline 60 & $(002253110 \ldots)$ & 14 & 380894 & 0.00152 \\
\hline
\end{tabular}

\begin{tabular}{|c|c|c|c|c|c|c|c|c|c|}
\hline & $p$-vector & $F$ & $N$ & $f$ & & $p$-vector & $F$ & $N$ & $f$ \\
\hline 61 & $(001532210 \ldots)$ & 14 & 380290 & 0.00152 & 121 & $(001364110 \ldots)$ & 16 & 255752 & 0.00102 \\
\hline 62 & $(000363000 \ldots)$ & 12 & 378639 & 0.00152 & 122 & $(000451100 \ldots)$ & 11 & 255748 & 0.00102 \\
\hline 63 & $(000444000 \ldots)$ & 12 & 369036 & 0.00148 & 123 & $(001523300 \ldots)$ & 14 & 255714 & 0.00102 \\
\hline 64 & $(002341210 \ldots)$ & 13 & 365352 & 0.00146 & 124 & $(001353010 \ldots)$ & 13 & 253371 & 0.00101 \\
\hline 65 & $(002424210 \ldots)$ & 15 & 363294 & 0.00145 & 125 & $(002434310 \ldots)$ & 17 & 253316 & 0.00101 \\
\hline 66 & $(003223110 \ldots)$ & 12 & 360507 & 0.00144 & 126 & $(000440000 \ldots)$ & 8 & 252807 & 0.00101 \\
\hline 67 & $(002331300 \ldots)$ & 12 & 360474 & 0.00144 & 127 & $(003313210 \ldots)$ & 13 & 252543 & 0.00101 \\
\hline 68 & $(002433310 \ldots)$ & 16 & 359007 & 0.00144 & 128 & $(003322310 \ldots)$ & 14 & 252509 & 0.00101 \\
\hline 69 & $(001253000 \ldots)$ & 11 & 343540 & 0.00137 & 129 & $(001434010 \ldots)$ & 13 & 252496 & 0.00101 \\
\hline 70 & $(001363110 \ldots)$ & 15 & 342759 & 0.00137 & 130 & $(002325200 \ldots)$ & 14 & 251827 & 0.00101 \\
\hline 71 & $(002224000 \ldots)$ & 10 & 342223 & 0.00137 & 131 & $(000372100 \ldots)$ & 13 & 251247 & 0.00101 \\
\hline 72 & $(002252110 \ldots)$ & 13 & 338813 & 0.00136 & 132 & $(002252300 \ldots)$ & 14 & 248444 & 0.00099 \\
\hline 73 & $(001261100 \ldots)$ & 11 & 337744 & 0.00135 & 133 & $(002431310 \ldots)$ & 14 & 246295 & 0.00099 \\
\hline 74 & $(001435200 \ldots)$ & 15 & 337609 & 0.00135 & 134 & $(002253300 \ldots)$ & 15 & 245427 & 0.00098 \\
\hline 75 & $(000532100 \ldots)$ & 11 & 335062 & 0.00134 & 135 & $(002443220 \ldots)$ & 17 & 244899 & .00098 \\
\hline 76 & $(001421100 \ldots)$ & 9 & 326573 & 0.00131 & 136 & $(003123100 \ldots)$ & 10 & 244466 & 0.00098 \\
\hline 77 & $(001441300 \ldots)$ & 13 & 324983 & 0.00130 & 137 & $(001513200 \ldots)$ & 12 & 244121 & 0.00098 \\
\hline 78 & $(002334300 \ldots)$ & 15 & 324192 & 0.00130 & 138 & $(001354010 \ldots)$ & 14 & 242450 & 0.00097 \\
\hline 79 & $(000542200 \ldots)$ & 13 & 321311 & 0.00129 & 139 & $(001543310 \ldots)$ & 17 & 242275 & 0.00097 \\
\hline 80 & $(001254000 \ldots)$ & 12 & 320972 & 0.00128 & 140 & $(001335000 \ldots)$ & 12 & 240198 & 0.00096 \\
\hline 81 & $(001264100 \ldots)$ & 14 & 317806 & 0.00127 & 141 & $(001352010 \ldots)$ & 12 & 239410 & 0.00096 \\
\hline 82 & $(001534210 \ldots)$ & 16 & 317202 & 0.00127 & 142 & $(002243010 \ldots)$ & 12 & 238926 & 0.00096 \\
\hline 83 & $(001444300 \ldots)$ & 16 & 316502 & 0.00127 & 143 & $(003323310 \ldots)$ & 15 & 238132 & 0.00095 \\
\hline 84 & $(000364000 \ldots)$ & 13 & 316407 & 0.00127 & 144 & $(002345210 \ldots)$ & 17 & 235822 & 0.00094 \\
\hline 85 & $(000543200 \ldots)$ & 14 & 315810 & 0.00126 & 145 & $(003223300 \ldots)$ & 13 & 235583 & 0.00094 \\
\hline 86 & $(000362000 \ldots)$ & 11 & 315678 & 0.00126 & 146 & $(000373100 \ldots)$ & 14 & 235216 & 0.00094 \\
\hline 87 & $(001425100 \ldots)$ & 13 & 315177 & 0.00126 & 147 & $(003222110 \ldots)$ & 11 & 234520 & 0.00094 \\
\hline 88 & $(002433120 \ldots)$ & 15 & 311076 & 0.00124 & 148 & $(001362300 \ldots)$ & 15 & 233130 & 0.00093 \\
\hline 89 & $(001454210 \ldots)$ & 17 & 308362 & 0.00123 & 149 & $(000455100 \ldots)$ & 15 & 232498 & 0.00093 \\
\hline 90 & $(001523110 \ldots)$ & 13 & 308320 & 0.00123 & 150 & $(003242310 \ldots)$ & 15 & 231928 & 0.00093 \\
\hline 91 & $(002254110 \ldots)$ & 15 & 300175 & 0.00120 & 151 & $(002262210 \ldots)$ & 15 & 231609 & 0.00093 \\
\hline 92 & $(002335110 \ldots)$ & 15 & 297450 & 0.00119 & 152 & $(003224110 \ldots)$ & 13 & 230872 & 0.00092 \\
\hline 93 & $(001524110 \ldots)$ & 14 & 297275 & 0.00119 & 153 & $(003212200 \ldots)$ & 10 & 230605 & 0.00092 \\
\hline 94 & $(000462200 \ldots)$ & 14 & 296941 & 0.00119 & 154 & $(003333220 \ldots)$ & 16 & 229635 & 0.00092 \\
\hline 95 & $(001441110 \ldots)$ & 12 & 296622 & 0.00119 & 155 & $(003231210 \ldots)$ & 12 & 228407 & 0.00091 \\
\hline 96 & $(001362110 \ldots)$ & 14 & 295553 & 0.00118 & 156 & $(001363300 \ldots)$ & 16 & 227814 & 0.00091 \\
\hline 97 & $(000441000 \ldots)$ & 9 & 291934 & 0.00117 & 157 & $(001251000 \ldots)$ & 9 & 227684 & 0.00091 \\
\hline 98 & $(002331110 \ldots)$ & 11 & 285885 & 0.00114 & 158 & $(002245200 \ldots)$ & 15 & 226914 & 0.00091 \\
\hline 99 & $(002432120 \ldots)$ & 14 & 284740 & 0.00114 & 159 & $(001514200 \ldots)$ & 13 & 226510 & 0.00091 \\
\hline 100 & $(002352310 \ldots)$ & 16 & 281706 & 0.00113 & 160 & $(002314100 \ldots)$ & 11 & 226362 & 0.00091 \\
\hline 101 & $(002241200 \ldots)$ & 11 & 280656 & 0.00112 & 161 & $(000522000 \ldots)$ & 9 & 223821 & 0.00090 \\
\hline 102 & $(001445110 \ldots)$ & 16 & 280426 & 0.00112 & 162 & $(002352120 \ldots)$ & 15 & 222637 & 0.00089 \\
\hline 103 & $(001355200 \ldots)$ & 16 & 279018 & 0.00112 & 163 & $(002313100 \ldots)$ & 10 & 222542 & 0.00089 \\
\hline 104 & $(001522110 \ldots)$ & 12 & 278044 & 0.00111 & 164 & $(002263210 \ldots)$ & 16 & 222046 & 0.00089 \\
\hline 105 & $(001451210 \ldots)$ & 14 & 275887 & 0.00110 & 165 & $(003323120 \ldots)$ & 14 & 221973 & 0.00089 \\
\hline 106 & $(002421210 \ldots)$ & 12 & 275389 & 0.00110 & 166 & $(003132200 \ldots)$ & 11 & 219674 & 0.00088 \\
\hline 107 & $(002235100 \ldots)$ & 13 & 275247 & 0.00110 & 167 & $(000445000 \ldots)$ & 13 & 218836 & 0.00088 \\
\hline 108 & $(003312210 \ldots)$ & 12 & 272966 & 0.00109 & 168 & $(003222300 \ldots)$ & 12 & 218196 & 0.00087 \\
\hline 109 & $(000463200 \ldots)$ & 15 & 272633 & 0.00109 & 169 & $(002353120 \ldots)$ & 16 & 217689 & 0.00087 \\
\hline 110 & $(002231100 \ldots)$ & 9 & 269118 & 0.00108 & 170 & $(002433201 \ldots)$ & 15 & 217182 & 0.00087 \\
\hline 111 & $(000544200 \ldots)$ & 15 & 266645 & 0.00107 & 171 & $(002244010 \ldots)$ & 13 & 212408 & 0.00085 \\
\hline 112 & $(001433010 \ldots)$ & 12 & 265340 & 0.00106 & 172 & $(001531210 \ldots)$ & 13 & 212259 & 0.00085 \\
\hline 113 & $(003234210 \ldots)$ & 15 & 264604 & 0.00106 & 173 & $(002432201 \ldots)$ & 14 & 211664 & 0.00085 \\
\hline 114 & $(002242010 \ldots)$ & 11 & 264147 & 0.00106 & 174 & $(002434120 \ldots)$ & 16 & 210983 & 0.00084 \\
\hline 115 & $(002442220 \ldots)$ & 16 & 264107 & 0.00106 & 175 & $(001346100 \ldots)$ & 15 & 210485 & 0.00084 \\
\hline 116 & $(001512200 \ldots)$ & 11 & 263778 & 0.00106 & 176 & $(003143110 \ldots)$ & 13 & 210362 & 0.00084 \\
\hline 117 & $(001431200 \ldots)$ & 11 & 261858 & 0.00105 & 177 & $(002324010 \ldots)$ & 12 & 210247 & 0.00084 \\
\hline 118 & $(002353310 \ldots)$ & 17 & 257245 & 0.00103 & 178 & $(002251300 \ldots)$ & 13 & 210141 & 0.00084 \\
\hline 119 & $(003332220 \ldots)$ & 15 & 256849 & 0.00103 & 179 & $(000464200 \ldots)$ & 16 & 209003 & 0.00084 \\
\hline 120 & $(001542310 \ldots)$ & 16 & 255888 & 0.00102 & 180 & $(001522300 \ldots)$ & 13 & 208541 & 0.00083 \\
\hline
\end{tabular}

TABLE II. The 180 most frequent $p$-vectors in the Poisson-Voronoi and grain growth microstructures. $F$ indicates the number of faces, $N$ indicates the absolute frequency, and $f$ indicates the relative frequency. A list of all $p$-vectors found in the data set along with frequencies can be found in the file all_pvectors.data. 


\begin{tabular}{|c|c|c|c|c|c|c|}
\hline & Weinberg vector & $F$ & $p$-vector & $S$ & $\bar{N}$ & $f$ \\
\hline 1 & ABCACDEFAFGHIBIJKDKLELMGMNHNJNMLKJIHGFEDCB & 9 & $(00133200 \ldots)$ & 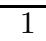 & 686096 & 0.00274 \\
\hline 2 & ABCACDEFAFGHBHIJDJKEKLGLILKJIHGFEDCB & 8 & $(00133100 \ldots)$ & 2 & 414904 & 0.00166 \\
\hline 3 & ABCDADEFAFGHIBIJKCKLMEMNGNOHOPJPLPONMLKJIHGFEDCB & 10 & $(00044200 \ldots)$ & 2 & 394197 & 0.00158 \\
\hline 4 & ABCACDEFAFGHIBIJKLDLMEMNGNOPHPJPOKONMLKJIHGFEDCB & 10 & $(00134110 \ldots)$ & 1 & 300751 & 0.00120 \\
\hline 5 & ABCDADEFAFGHBHIJCJKLELMGMNINKNMLKJIHGFEDCB & 9 & $(00044100 \ldots)$ & 4 & 291934 & 0.00117 \\
\hline 6 & ABCDADEFAFGHBHIJCJKEKLGLILKJIHGFEDCB & 8 & $(00044000 \ldots)$ & 8 & 252807 & 0.00101 \\
\hline 7 & ABCACDEFAFGHIBIJKLDLMEMNOGOPHPJPONKNMLKJIHGFEDCB & 10 & $(00142210 \ldots)$ & 1 & 239579 & 0.00096 \\
\hline 8 & ABCDADEFGAGHIBIJKCKLMEMNFNOPHPQJQRLRORQPONMLKJIH & 11 & $(00036200 \ldots)$ & 2 & 237965 & 0.00095 \\
\hline 9 & ABCACDEFAFGHIBIJKDKLELMNGNOHOPJPMPONMLKJIHGFEDCB & 10 & $(00133300 \ldots)$ & 1 & 236692 & 0.00095 \\
\hline 10 & ABCDADEFAFGHBHIJKCKLMEMNOGOPIPQJQRLRNRQPONMLKJIH & 11 & $(00044300 \ldots)$ & 1 & 234533 & 0.00094 \\
\hline 11 & ABCACDEFAFGHIBIJKLDLMNENGNMOPHPJPOKOMLKJIHGFEDCB & 10 & $(00142210 \ldots)$ & 1 & 234180 & 0.00094 \\
\hline 12 & ABCACDEFGAGHIJBJKLMDMNENOPFPHPOQRIRKRQLQONMLKJIH & 11 & $(00142310 \ldots)$ & 1 & 233523 & 0.00093 \\
\hline 13 & ABCDADEFAFGHBHIJCJKLELMNGNOIOPKPMPONMLKJIHGFEDCB & 10 & $(00044200 \ldots)$ & 2 & 227853 & 0.00091 \\
\hline 14 & ABCACDEFAFGHBHIJDJKLELMGMNINKNMLKJIHGFEDCB & 9 & $(00125100 \ldots)$ & 2 & 227684 & 0.00091 \\
\hline 15 & ABCDADEFAFGHBHIJKCKLELMNGNINMJMLKJIHGFEDCB & 9 & $(00052200 \ldots)$ & 4 & 223821 & 0.00090 \\
\hline 16 & ABCACDEFAFGHIBIJKDKLMEMNGNOPHPJPOLONMLKJIHGFEDCB & 10 & $(00125200 \ldots)$ & 2 & 219647 & 0.00088 \\
\hline 17 & ABCACDEFAFGHIBIJKDKLMEMNGNOHOPJPLPONMLKJIHGFEDCB & 10 & $(00125200 \ldots)$ & 2 & 204714 & 0.00082 \\
\hline 18 & ABCACDEFAFGHIBIJKLDLMNENOGOPQHQJQPRKRMRPONMLKJIH & 11 & $(00126110 \ldots)$ & 1 & 204666 & 0.00082 \\
\hline 19 & ABCACDEFAFGHIBIJKLDLMNENOGOPKPMPONMLKJHJIHGFEDCB & 10 & $(00223210 \ldots)$ & 1 & 202792 & 0.00081 \\
\hline 20 & ABCACDEFAFGHIBIJKLDLMEMNGNKNMLKJHJIHGFEDCB & 9 & $(00223110 \ldots)$ & 2 & 200420 & 0.00080 \\
\hline 21 & ABCDADEFGAGHIBIJKCKLMEMNFNOHOPJPLPONMLKJIHGFEDCB & 10 & $(00036100 \ldots)$ & 6 & 196335 & 0.00079 \\
\hline 22 & ABCACDEFGAGHIJBJKLDLMEMNFNOHOPIPKPONMLKJIHGFEDCB & 10 & $(00141400 \ldots)$ & 2 & 192647 & 0.00077 \\
\hline 23 & ABCDADEFAFGHIBIJKCKLMEMNOGOPHPQJQRLRNRQPONMLKJIH & 11 & $(00044300 \ldots)$ & 2 & 188196 & 0.00075 \\
\hline 24 & ABCACDEFAFGHIJBJKLMDMNENOPGPHPOLONMLKIKJIHGFEDCB & 10 & $(00321220 \ldots)$ & 1 & 182319 & 0.00073 \\
\hline 25 & ABCDADEFGAGHIBIJKCKLELMFMNHNJNMLKJIHGFEDCB & 9 & $(00036000 \ldots)$ & 12 & 175908 & 0.00070 \\
\hline 26 & ABCACDEFAFGHIBIJKDKLELGLKJHJIHGFEDCB & 8 & $(00222200 \ldots)$ & 4 & 172020 & 0.00069 \\
\hline 27 & ABCACDEFAFGHIBIJKLDLMNENGNMKMLKJHJIHGFEDCB & 9 & $(00312210 \ldots)$ & 2 & 167986 & 0.00067 \\
\hline 28 & ABCDADEFGAGHIBIJKCKLMEMNOFOPQHQRJRSTLTNTSPSRQPON & 12 & $(00036300 \ldots)$ & 1 & 167251 & 0.00067 \\
\hline 29 & ABCACDEFAFGHIBIJKLDLMEMNGNOHOPJPKPONMLKJIHGFEDCB & 10 & $(00223210 \ldots)$ & 1 & 167141 & 0.00067 \\
\hline 30 & ABCACDEFAFGHBHIJKDKLELMNGNINMJMLKJIHGFEDCB & 9 & $(00142110 \ldots)$ & 2 & 166543 & 0.00067 \\
\hline 31 & ABCDADEFAFGHBHIJCJKLMEMNGNOPIPKPOLONMLKJIHGFEDCB & 10 & $(00053110 \ldots)$ & 2 & 165628 & 0.00066 \\
\hline 32 & ABCACDEFAFGHIBIJKDKLMEMNOGOPHPQJQRLRNRQPONMLKJIH & 11 & $(00125300 \ldots)$ & 1 & 159324 & 0.00064 \\
\hline 33 & ABCACDEFGAGHIJBJKLMDMEMLNOFOHONPIPKPNLKJIHGFEDCB & 10 & $(00231310 \ldots)$ & 2 & 158213 & 0.00063 \\
\hline 34 & ABCACDEFAFGHBHIDIJEJGJIHGFEDCB & 7 & $(00133000 \ldots)$ & 6 & 154332 & 0.00062 \\
\hline 35 & ABCACDEAEFGHBHIJKDKLFLJLKJIGIHGFEDCB & 8 & $(00312110 \ldots)$ & 1 & 150240 & 0.00060 \\
\hline 36 & ABCDADEFAFGHIBIJKCKLMNENOGOPQHQRJRLRQPMPONMLKJIH & 11 & $(00045110 \ldots)$ & 2 & 150040 & 0.00060 \\
\hline 37 & ABCACDEFAFGHBHIJKDKLELMNGNOIOPJPMPONMLKJIHGFEDCB & 10 & $(00134110 \ldots)$ & 1 & 148303 & 0.00059 \\
\hline 38 & ABCACDEFAFGHIBIJKLDLMNENOGOPQRHRJRQKQPMPONMLKJIH & 11 & $(00134210 \ldots)$ & 1 & 145835 & 0.00058 \\
\hline 39 & ABCDADEFGAGHIBIJKCKLMEMNOFOPHPQJQRLRNRQPONMLKJIH & 11 & $(00028100 \ldots)$ & 4 & 144758 & 0.00058 \\
\hline 40 & ABCACDEFAFGHIBIJKLDLMEMNOGOPQHQJQPRKRNRPONMLKJIH & 11 & $(00134210 \ldots)$ & 1 & 137500 & 0.00055 \\
\hline 41 & ABCACDEFAFGHIBIJKDKLELMNGNHNMJMLKJIHGFEDCB & 9 & $(00222300 \ldots)$ & 2 & 135758 & 0.00054 \\
\hline 42 & ABCACDEFGAGHIJBJKLMDMNENOFOPHPQRIRKRQLQPONMLKJIH & 11 & $(00142310 \ldots)$ & 1 & 133849 & 0.00054 \\
\hline 43 & ABCACDEAEFGHBHIJKDKLFLMNGNINMJMLKJIHGFEDCB & 9 & $(00142110 \ldots)$ & 1 & 132858 & 0.00053 \\
\hline 44 & ABCACDEFGAGHIJBJKLDLMEMNOFOPHPQRIRKRQNQPONMLKJIH & 11 & $(00133400 \ldots)$ & 2 & 132080 & 0.00053 \\
\hline 45 & ABCACDEFGAGHIJBJKLDLMEMNOFOPHPQIQRKRNRQPONMLKJIH & 11 & $(00125300 \ldots)$ & 2 & 131980 & 0.00053 \\
\hline 46 & ABCACDEFAFGHIBIJKLDLMNENOGOPHPQJQRKRMRQPONMLKJIH & 11 & $(00134210 \ldots)$ & 1 & 130636 & 0.00052 \\
\hline 47 & ABCACDEFAFGHIBIJKDKLMEMNOGOHONPJPLPNMLKJIHGFEDCB & 10 & $(00222400 \ldots)$ & 1 & 130506 & 0.00052 \\
\hline 48 & ABCDADEFAFGHBHIJCJKLMEMNOGOPQIQKQPRLRNRPONMLKJIH & 11 & $(00053210 \ldots)$ & 1 & 129216 & 0.00052 \\
\hline 49 & ABCACDEFAFGHIBIJKLDLMEMNOGOPHPQJQRKRNRQPONMLKJIH & 11 & $(00134210 \ldots)$ & 1 & 127830 & 0.00051 \\
\hline 50 & ABCACDEFGAGHIJBJKLMDMEMLNOFOPHPQIQRKRNRQPONLKJIH & 11 & $(00223310 \ldots)$ & 1 & 125216 & 0.00050 \\
\hline
\end{tabular}

TABLE III. The 100 most common Weinberg vectors and their number of faces $F$, $p$-vector, the order $S$ of the associated symmetry group, and frequencies $f$ in the Poisson-Voronoi microstructure. To consolidate the data, integers are replaced with letters (e.g., 1 is replaced with A, 2 with B, etc.). Long Weinberg vectors are truncated in a manner that leaves the truncated part unambiguous. The 10,000 most common Weinberg vectors can be found in the file top_10000_wvectors.data; the entire list of all 72,101,233 observed Weinberg vectors is too large to include. 


\begin{tabular}{|c|c|c|c|c|c|c|}
\hline & Weinberg vector & $F$ & $p$-vector & $S$ & $N$ & $f$ \\
\hline 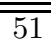 & ABCACDEFGAGHIJBJKLDLELKMNFNHNMIMKJIHGFEDCB & 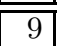 & $(00230400 \ldots)$ & 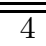 & 124995 & 0.00050 \\
\hline 52 & ABCACDEFAFGHIJBJKLMDMNENOGOPHPLPONMLKIKJIHGFEDCB & 10 & $(00224020 \ldots)$ & 2 & 124137 & 0.00050 \\
\hline 53 & ABCDADEFAFGHBHIJKCKLMEMNOGOIONPJPLPNMLKJIHGFEDCB & 10 & $(00052300 \ldots)$ & 4 & 124069 & 0.00050 \\
\hline 54 & ABCACDEAEFGHBHIJDJKFKLGLILKJIHGFEDCB & 8 & $(00141200 \ldots)$ & 2 & 123938 & 0.00050 \\
\hline 55 & ABCACDEAEFGHBHIJKDKLMFMNGNINMLJLKJIHGFEDCB & 9 & $(00231210 \ldots)$ & 1 & 122663 & 0.00049 \\
\hline 56 & ABCDADEFGAGHIBIJKLCLMNENOFOPQHQJQPRKRMRPONMLKJIH & 11 & $(00044300 \ldots)$ & 4 & 122348 & 0.00049 \\
\hline 57 & ABCACDEFAFGHIBIJKLDLMNOEOPGPQRHRJRQNQPONMKMLKJIH & 11 & $(00223310 \ldots)$ & 1 & 120472 & 0.00048 \\
\hline 58 & ABCDADEFAFGHIBIJKCKLMEMNOGOPHPQRJRLRQNQPONMLKJIH & 11 & $(00052400 \ldots)$ & 2 & 119443 & 0.00048 \\
\hline 59 & ABCDADEFAFGHIBIJKCKLMNENOPGPQHQRSJSLSRTMTOTRQPO & 12 & $(00053310 \ldots)$ & 1 & 117048 & 0.00047 \\
\hline 60 & ABCACDEFAFGHBHIJKDKLELMGMNINJNMLKJIHGFEDCB & 9 & $(00215010 \ldots)$ & 2 & 114530 & 0.00046 \\
\hline 61 & ABCACDEFGAGHIJBJKLDLMEMNOFOHONPIPKPNMLKJIHGFEDCB & 10 & $(00133300 \ldots)$ & 6 & 114298 & 0.00046 \\
\hline 62 & ABCACDEFAFGHIBIJKDKLMEMNOGOPQHQJQPRLRNRPONMLKJIH & 11 & $(00133400 \ldots)$ & 1 & 113239 & 0.00045 \\
\hline 63 & ABCACDEFAFGHBHIJKDKLMEMNGNOPIPJPOLONMLKJIHGFEDCB & 10 & $(00215110 \ldots)$ & 1 & 111236 & 0.00044 \\
\hline 64 & ABCACDEFAFGHIJBJKLMDMNENOGOPHPQRIRKRQLQPONMLKJIH & 11 & $(00143120 \ldots)$ & 1 & 110830 & 0.00044 \\
\hline 65 & ABCDADEFAFGHIBIJKCKLMNENOGOPHPQRJRLRQMQPONMLKJIH & 11 & $(00053210 \ldots)$ & 2 & 109614 & 0.00044 \\
\hline 66 & ABCACDEFAFGHIBIJKDKLMEMNOGOPHPQRJRLRQNQPONMLKJIH & 11 & $(00133400 \ldots)$ & 1 & 104620 & 0.00042 \\
\hline 67 & ABCDADEFAFGHIBIJKCKLMEMNOGOPHPQRJRSLSTNTQTSRQPO & 12 & $(00044400 \ldots)$ & 1 & 104459 & 0.00042 \\
\hline 68 & ABCACDEFAFGHBHIJDJKLELGLKIKJIHGFEDCB & 8 & $(00214100 \ldots)$ & 4 & 103424 & 0.00041 \\
\hline 69 & ABCDADEFGAGHIBIJKLCLMNENOPFPQHQRJRSKSTMTOTS & 12 & $(00036300 \ldots)$ & 2 & 103145 & 0.00041 \\
\hline 70 & ABCACDEFAFGHBHIJDJKEKLMGMNINLNMLKJIHGFEDCB & 9 & $(00214200 \ldots)$ & 2 & 101630 & 0.00041 \\
\hline 71 & ABCACDEFGAGHIJBJKLDLMEMNFNOPHPQIQRKRORQPONMLKJIH & 11 & $(00141500 \ldots)$ & 1 & 100197 & 0.00040 \\
\hline 72 & ABCACDEFAFGHIJBJKLMDMNENOGOPQHQRIRKRQPLPONMLKJIH & 11 & $(00135020 \ldots)$ & 1 & 99613 & 0.00040 \\
\hline 73 & ABCACDEFGAGHIJBJKLMDMNENOPQRFRHRQIQPKPOLONMLKJIH & 11 & $(00151220 \ldots)$ & 1 & 98188 & 0.00039 \\
\hline 74 & ABCACDEFGAGHIJBJKLMDMNENOPQFQHQPRIRSKSTLTOTSRPO & 12 & $(00134310 \ldots)$ & 1 & 96027 & 0.00038 \\
\hline 75 & ABCACDEFAFGHIJBJKLMDMNENOPGPQHQRLRORQPONMLKIKJIH & 11 & $(00232220 \ldots)$ & 1 & 95947 & 0.00038 \\
\hline 76 & ABCDADEFGAGHIBIJKLCLMNENOFOPQHQRJRSTKTMTSPSRQPO & 12 & $(00044400 \ldots)$ & 2 & 95423 & 0.00038 \\
\hline 77 & ABCACDEFGAGHIJBJKLMDMNENOPFPQHQRIRSKSTLTOTSRQPO & 12 & $(00134310 \ldots)$ & 1 & 94607 & 0.00038 \\
\hline 78 & ABCACDEFAFGHBHIJDJKLELMNGNOPIPKPOMONMLKJIHGFEDCB & 10 & $(00214300 \ldots)$ & 1 & 94178 & 0.00038 \\
\hline 79 & ABCACDEFAFGHIBIJKLDLMNENOPGPQHQJQPORKRMRONMLKJIH & 11 & $2310 \ldots)$ & 1 & 92974 & 0.00037 \\
\hline 80 & ABCACDEFGAGHIJBJKLMDMEMLNOPFPHPOQIQRKRNRQONLKJIH & 11 & $(00232220 \ldots)$ & 1 & 92593 & 0.00037 \\
\hline 81 & ABCACDEFGAGHIJBJKLDLMEMNOFOPHPQRIRSKSTNTQTSRQPO & 12 & $(00125400 \ldots)$ & 1 & 92511 & 0.00037 \\
\hline 82 & ABCACDEFAFGHIBIJKLDLMNENOGOPHPJPONMKMLKJIHGFEDCB & 10 & $(00223210 \ldots)$ & 2 & 92455 & 0.00037 \\
\hline 83 & ABCDADEFAFGHBHIJCJKLELMNOGOPIPQRKRMRQNQPONMLKJIH & 11 & $(00053210 \ldots)$ & 1 & 92066 & 0.00037 \\
\hline 84 & ABCACDEFAFGHBHIJKDKLMEMNGNOIOPJPLPONMLKJIHGFEDCB & 10 & $(00126010 \ldots)$ & 2 & 91831 & 0.00037 \\
\hline 85 & ABCDADEFAFGHBHICIJEJGJIHGFEDCB & 7 & $(00052000 \ldots)$ & 20 & 91654 & 0.00037 \\
\hline 86 & ABCDADEFAFGHIBIJKCKLMNENOGOPQHQRSJSLSRTMTPTRQPO & 12 & $(00045210 \ldots)$ & 1 & 91577 & 0.00037 \\
\hline 87 & ABCDADEFGAGHIBIJKCKLMEMNOFOPHPQRJRSLSTNTQTSRQPO & 12 & $(00028200 \ldots)$ & 2 & 90317 & 0.00036 \\
\hline 88 & ABCACDEFAFGHBHIJKDKLELMNGNOPIPJPOMONMLKJIHGFEDCB & 10 & $(00312310 \ldots)$ & 1 & 86892 & 0.00035 \\
\hline 89 & ABCACDEFAFGHBHIJDJKLMEMNGNLNMLKIKJIHGFEDCB & 9 & $(00303300 \ldots)$ & 2 & 86215 & 0.00034 \\
\hline 90 & ABCACDEFAFGHIJBJKLMDMNOEOPGPQHQRLRNRQPONMLKIKJIH & 11 & $(00224120 \ldots)$ & 2 & 85589 & 0.00034 \\
\hline 91 & ABCACDEFAFGHBHIJKDKLMEMNOGOPIPQJQRLRNRQPONMLKJIH & 11 & $(00126110 \ldots)$ & 1 & 85417 & 0.00034 \\
\hline 92 & ABCACDEFAFGHIBIJKLDLMNOEOGONPQHQJQPRKRMRPNMLKJIH & 11 & $(00142310 \ldots)$ & 1 & 84838 & 0.00034 \\
\hline 93 & ABCACDEFAFGHIJBJKLMDMNENOPGPHPOQRIRKRQLQONMLKJIH & 11 & $(00232220 \ldots)$ & 1 & 84609 & 0.00034 \\
\hline 94 & ABCACDEFGAGHIJBJKLMDMNENOPFPQHQRSTITKTSLSRORQPO & 12 & $(00142410 \ldots)$ & 1 & 83475 & 0.00033 \\
\hline 95 & ABCACDEFAFGHIBIJKDKLMEMNGNOPHPQJQRLRORQPONMLKJIH & 11 & $(00117200 \ldots)$ & 2 & 82555 & 0.00033 \\
\hline 96 & ABCACDEFAFGHIJBJKLMDMNOEOPQGQHQPRLRNRPONMLKIKJIH & 11 & $(00322130 \ldots)$ & 1 & 81394 & 0.00033 \\
\hline 97 & ABCACDEAEFGHIBIJKLDLMFMNGNOPHPJPOKONMLKJIHGFEDCB & 10 & $(00143020 \ldots)$ & 1 & 81141 & 0.00032 \\
\hline 98 & ABCACDEFAFGHIBIJKDKLELMNOGOHONPJPMPNMLKJIHGFEDCB & 10 & $(00312310 \ldots)$ & 1 & 81045 & 0.00032 \\
\hline 99 & ABCACDEFAFGHIJBJKLMNDNOEOPGPQHQRLRMRQPONMLKIKJIH & 11 & $(00322211 \ldots)$ & 1 & 81022 & 0.00032 \\
\hline 100 & ABCDADEFAFGHIJBJKCKLMNENOGOPHPQRIRLRQMQPONMLKJIH & 11 & $(00054020 \ldots)$ & 2 & 80250 & 0.00032 \\
\hline
\end{tabular}

TABLE IV. The 100 most common Weinberg vectors and their number of faces $F$, $p$-vector, the order $S$ of the associated symmetry group, and frequencies $f$ in the Poisson-Voronoi microstructure. To consolidate the data, integers are replaced with letters (e.g., 1 is replaced with A, 2 with B, etc.). Long Weinberg vectors are truncated in a manner that leaves the truncated part unambiguous. The 10,000 most common Weinberg vectors can be found in the file top_10000_wvectors.data; the entire list of all 72,101,233 observed Weinberg vectors is too large to include. 


\begin{tabular}{|c|r|c|c|c|}
\hline$n$ & \multicolumn{1}{|c|}{$N$} & $f$ & $\left\langle x_{a}\right\rangle_{n}$ & $\left\langle x_{l}\right\rangle_{n}$ \\
\hline 1 & 0 & 0.0000 & $0.0000 \pm 0.0000$ & $0.0000 \pm 0.0000$ \\
2 & 0 & 0.0000 & $0.0000 \pm 0.0000$ & $0.0000 \pm 0.0000$ \\
3 & 261661169 & 0.1347 & $0.0928 \pm 0.1595$ & $1.7435 \pm 1.4534$ \\
4 & 446276118 & 0.2298 & $0.3651 \pm 0.3952$ & $3.5491 \pm 1.8703$ \\
5 & 469146641 & 0.2416 & $0.8115 \pm 0.6274$ & $5.1951 \pm 1.9856$ \\
6 & 369632159 & 0.1903 & $1.3458 \pm 0.7981$ & $6.5723 \pm 1.9352$ \\
7 & 226308795 & 0.1165 & $1.8932 \pm 0.9062$ & $7.6811 \pm 1.8219$ \\
8 & 109221807 & 0.0562 & $2.4178 \pm 0.9727$ & $8.5766 \pm 1.7028$ \\
9 & 42138921 & 0.0217 & $2.9102 \pm 1.0157$ & $9.3172 \pm 1.5987$ \\
10 & 13206845 & 0.0068 & $3.3708 \pm 1.0463$ & $9.9465 \pm 1.5134$ \\
11 & 3419199 & 0.0018 & $3.8028 \pm 1.0698$ & $10.4938 \pm 1.4443$ \\
12 & 738992 & 0.0004 & $4.2134 \pm 1.0887$ & $10.9834 \pm 1.3875$ \\
13 & 136155 & 0.0001 & $4.6086 \pm 1.1078$ & $11.4328 \pm 1.3433$ \\
14 & 21470 & 0.0000 & $4.9779 \pm 1.1227$ & $11.8333 \pm 1.3061$ \\
15 & 2962 & 0.0000 & $5.3197 \pm 1.1298$ & $12.1899 \pm 1.2624$ \\
16 & 375 & 0.0000 & $5.7022 \pm 1.1513$ & $12.5823 \pm 1.2147$ \\
17 & 52 & 0.0000 & $6.2794 \pm 1.1861$ & $13.1853 \pm 1.1916$ \\
18 & 5 & 0.0000 & $5.6993 \pm 1.4205$ & $12.2969 \pm 1.6639$ \\
19 & 0 & 0.0000 & $0.0000 \pm 0.0000$ & $0.0000 \pm 0.0000$ \\
20 & 0 & 0.0000 & $0.0000 \pm 0.0000$ & $0.0000 \pm 0.0000$ \\
\hline
\end{tabular}

TABLE V. Faces of different topological types; a total of 1,941,911,665 faces. Data included are number of edges $n$, absolute frequency $N$, relative frequency $f$, average normalized area $\left\langle x_{a}\right\rangle_{n}$, and average normalized perimeter $\left\langle x_{l}\right\rangle_{n}$. Included for area and perimeter is standard deviation from the mean. Areas are normalized so their mean is 1; perimeters are measured in units of average edge lengths. All data is included in the file edges_dist_areas_perims.data.

\begin{tabular}{|r|r|c|c|c|r|}
\hline$F$ & \multicolumn{1}{|c|}{$N$} & $f$ & $\left\langle x_{v}\right\rangle_{F}$ & $\left\langle x_{s}\right\rangle_{F}$ & $m(F)$ \\
\hline 1 & 0 & 0.000 & $0.000 \pm 0.000$ & $0.000 \pm 0.000$ & 0.000 \\
2 & 0 & 0.000 & $0.000 \pm 0.000$ & $0.000 \pm 0.000$ & 0.000 \\
3 & 0 & 0.000 & $0.000 \pm 0.000$ & $0.000 \pm 0.000$ & 0.000 \\
4 & 325 & 0.000 & $0.088 \pm 0.049$ & $0.255 \pm 0.089$ & 15.187 \\
5 & 9517 & 0.000 & $0.147 \pm 0.076$ & $0.344 \pm 0.111$ & 15.701 \\
6 & 86847 & 0.000 & $0.212 \pm 0.100$ & $0.422 \pm 0.124$ & 15.981 \\
7 & 434884 & 0.002 & $0.279 \pm 0.122$ & $0.493 \pm 0.133$ & 16.125 \\
8 & 1474073 & 0.006 & $0.352 \pm 0.144$ & $0.560 \pm 0.141$ & 16.218 \\
9 & 3757407 & 0.015 & $0.428 \pm 0.164$ & $0.625 \pm 0.148$ & 16.279 \\
10 & 7673585 & 0.031 & $0.506 \pm 0.184$ & $0.687 \pm 0.154$ & 16.311 \\
11 & 13105393 & 0.052 & $0.588 \pm 0.203$ & $0.748 \pm 0.160$ & 16.327 \\
12 & 19278192 & 0.077 & $0.673 \pm 0.221$ & $0.806 \pm 0.165$ & 16.329 \\
13 & 24913588 & 0.100 & $0.760 \pm 0.239$ & $0.864 \pm 0.169$ & 16.323 \\
14 & 28773718 & 0.115 & $0.849 \pm 0.257$ & $0.921 \pm 0.173$ & 16.310 \\
15 & 30069917 & 0.120 & $0.941 \pm 0.274$ & $0.976 \pm 0.177$ & 16.293 \\
16 & 28709465 & 0.115 & $1.034 \pm 0.291$ & $1.031 \pm 0.181$ & 16.272 \\
17 & 25258283 & 0.101 & $1.129 \pm 0.307$ & $1.084 \pm 0.185$ & 16.249 \\
18 & 20627759 & 0.083 & $1.225 \pm 0.323$ & $1.137 \pm 0.188$ & 16.224 \\
19 & 15733136 & 0.063 & $1.323 \pm 0.339$ & $1.188 \pm 0.191$ & 16.198 \\
20 & 11240712 & 0.045 & $1.422 \pm 0.354$ & $1.240 \pm 0.194$ & 16.171 \\
21 & 7575444 & 0.030 & $1.523 \pm 0.369$ & $1.290 \pm 0.197$ & 16.142 \\
\hline
\end{tabular}

\begin{tabular}{|r|r|c|c|c|c|}
\hline$F$ & \multicolumn{1}{|c|}{$N$} & $f$ & $\left\langle x_{v}\right\rangle_{F}$ & $\left\langle x_{s}\right\rangle_{F}$ & $m(F)$ \\
\hline 22 & 4821143 & 0.019 & $1.624 \pm 0.383$ & $1.340 \pm 0.200$ & 16.115 \\
23 & 2919932 & 0.012 & $1.727 \pm 0.397$ & $1.389 \pm 0.202$ & 16.086 \\
24 & 1678180 & 0.007 & $1.830 \pm 0.412$ & $1.437 \pm 0.205$ & 16.058 \\
25 & 919317 & 0.004 & $1.934 \pm 0.425$ & $1.484 \pm 0.207$ & 16.030 \\
26 & 482492 & 0.002 & $2.040 \pm 0.438$ & $1.532 \pm 0.209$ & 16.002 \\
27 & 243551 & 0.001 & $2.145 \pm 0.452$ & $1.578 \pm 0.212$ & 15.976 \\
28 & 116870 & 0.001 & $2.252 \pm 0.465$ & $1.624 \pm 0.213$ & 15.948 \\
29 & 54391 & 0.000 & $2.359 \pm 0.477$ & $1.669 \pm 0.215$ & 15.920 \\
30 & 24236 & 0.000 & $2.471 \pm 0.493$ & $1.717 \pm 0.219$ & 15.899 \\
31 & 10467 & 0.000 & $2.573 \pm 0.503$ & $1.758 \pm 0.219$ & 15.862 \\
32 & 4393 & 0.000 & $2.681 \pm 0.522$ & $1.802 \pm 0.224$ & 15.845 \\
33 & 1713 & 0.000 & $2.796 \pm 0.536$ & $1.848 \pm 0.228$ & 15.824 \\
34 & 643 & 0.000 & $2.918 \pm 0.537$ & $1.897 \pm 0.222$ & 15.796 \\
35 & 262 & 0.000 & $3.032 \pm 0.593$ & $1.935 \pm 0.244$ & 15.725 \\
36 & 104 & 0.000 & $3.133 \pm 0.569$ & $1.980 \pm 0.227$ & 15.702 \\
37 & 33 & 0.000 & $3.115 \pm 0.488$ & $1.963 \pm 0.198$ & 15.555 \\
38 & 14 & 0.000 & $3.266 \pm 0.601$ & $2.038 \pm 0.244$ & 15.455 \\
39 & 9 & 0.000 & $3.210 \pm 0.528$ & $2.006 \pm 0.211$ & 15.664 \\
40 & 3 & 0.000 & $3.696 \pm 0.666$ & $2.192 \pm 0.277$ & 15.692 \\
41 & 2 & 0.000 & $3.054 \pm 0.041$ & $1.953 \pm 0.045$ & 15.634 \\
42 & 0 & 0.000 & $0.000 \pm 0.000$ & $0.000 \pm 0.000$ & 0.000 \\
\hline
\end{tabular}

TABLE VI. Cells with different numbers of faces; a total of 250,000,000 cells. Data included are the absolute frequency $N$, the relative frequency $f$, average normalized volume $\left\langle x_{v}\right\rangle_{F}$, average normalized surface area $\left\langle x_{s}\right\rangle_{F}$, and average number of neighbors' neighbors $m(F)$. Included for volume and area is standard deviation from the mean. Volumes and surface areas normalized so means are 1. All data is included in the file faces_dist_vols_areas.data. 


\begin{tabular}{|r|r|r|r|r|}
\hline & \multicolumn{2}{|c|}{$p$-vectors } & \multicolumn{2}{|c|}{ Topological types } \\
\hline F & observed & possible & observed & possible \\
\hline 1 & 0 & 0 & 0 & 0 \\
2 & 0 & 0 & 0 & 0 \\
3 & 0 & 0 & 0 & 0 \\
4 & 1 & 1 & 1 & 1 \\
5 & 1 & 1 & 1 & 1 \\
6 & 2 & 2 & 2 & 2 \\
7 & 5 & 5 & 5 & 5 \\
8 & 13 & 13 & 14 & 14 \\
9 & 33 & 33 & 50 & 50 \\
10 & 85 & 85 & 233 & 233 \\
11 & 199 & 199 & 1249 & 1249 \\
12 & 440 & 445 & 7370 & 7595 \\
13 & 917 & 947 & 41,337 & 49,566 \\
14 & 1779 & 1909 & 204,367 & 339,722 \\
15 & 3254 & - & 864,084 & $2,406,841$ \\
16 & 5458 & - & $3,735,112$ & $17,490,241$ \\
17 & 8455 & - & $9,771,618$ & $129,664,753$ \\
18 & 12,417 & - & $13,858,582$ & $977,526,957$ \\
19 & 16,998 & - & $13,852,480$ & $7,475,907,149$ \\
20 & 22,267 & - & $10,944,266$ & $57,896,349,553$ \\
21 & 27,539 & - & $7,545,337$ & $453,382,272,049$ \\
\hline
\end{tabular}

\begin{tabular}{|c|r|r|r|r|}
\hline & \multicolumn{2}{|r|}{$p$-vectors } & \multicolumn{3}{|c|}{ Topological types } \\
\hline F & observed & possible & observed & \multicolumn{2}{|c|}{ possible } \\
\hline 22 & 32,295 & - & $4,818,687$ & $3,585,853,662,949$ \\
23 & 35,847 & - & $2,919,767$ & $28,615,703,421,545$ \\
24 & 37,821 & - & $1,678,171$ & - \\
25 & 37,327 & - & 919,317 & - \\
26 & 34,619 & - & 482,492 & - \\
27 & 30,142 & - & 243,551 & - \\
28 & 24,280 & - & 116,870 & - \\
29 & 17,879 & - & 54,391 & - \\
30 & 11,971 & - & 24,236 & - \\
31 & 7062 & - & 10,467 & - \\
32 & 3654 & - & 4393 & - \\
33 & 1590 & - & 1713 & - \\
34 & 634 & - & 643 & - \\
35 & 262 & - & 262 & - \\
36 & 103 & - & 104 & - \\
37 & 33 & - & 33 & - \\
38 & 14 & - & 14 & - \\
39 & 9 & - & 9 & - \\
40 & 3 & - & 3 & 2 \\
41 & 2 & - & - & \\
42 & 0 & - & & \\
\hline
\end{tabular}

TABLE VII. This table lists the number of distinct $p$-vectors and Weinberg vectors we observed in our data sets of $250,000,000$ cells. For each fixed number of faces $F$, we list both the number of observed $p$-vectors and Weinberg vectors, and also the total possible number of these. Data are obtained from [63]. There are a total of 375,410 distinct $p$-vectors in our data set; there are a total of 72,101,233 topological types. All data is included in the file p_vectors_and_types.data.

\begin{tabular}{|r|r|c|}
\hline$S$ & $N$ & $f$ \\
\hline 1 & 229260326 & 0.9170 \\
2 & 16524698 & 0.0661 \\
3 & 18385 & 0.0001 \\
4 & 2506544 & 0.0100 \\
6 & 705748 & 0.0028 \\
8 & 414776 & 0.0017 \\
10 & 914 & 0.0000 \\
12 & 283733 & 0.0011 \\
16 & 77449 & 0.0003 \\
20 & 91681 & 0.0004 \\
24 & 72273 & 0.0003 \\
28 & 12877 & 0.0000 \\
32 & 2261 & 0.0000 \\
36 & 292 & 0.0000 \\
40 & 21 & 0.0000 \\
44 & 2 & 0.0000 \\
48 & 24408 & 0.0000 \\
120 & 3612 & 0.0000 \\
\hline
\end{tabular}

TABLE VIII. Absolute and relative frequencies of grains with various symmetry group orders $S$. All data is included in the file symmetries.data. 


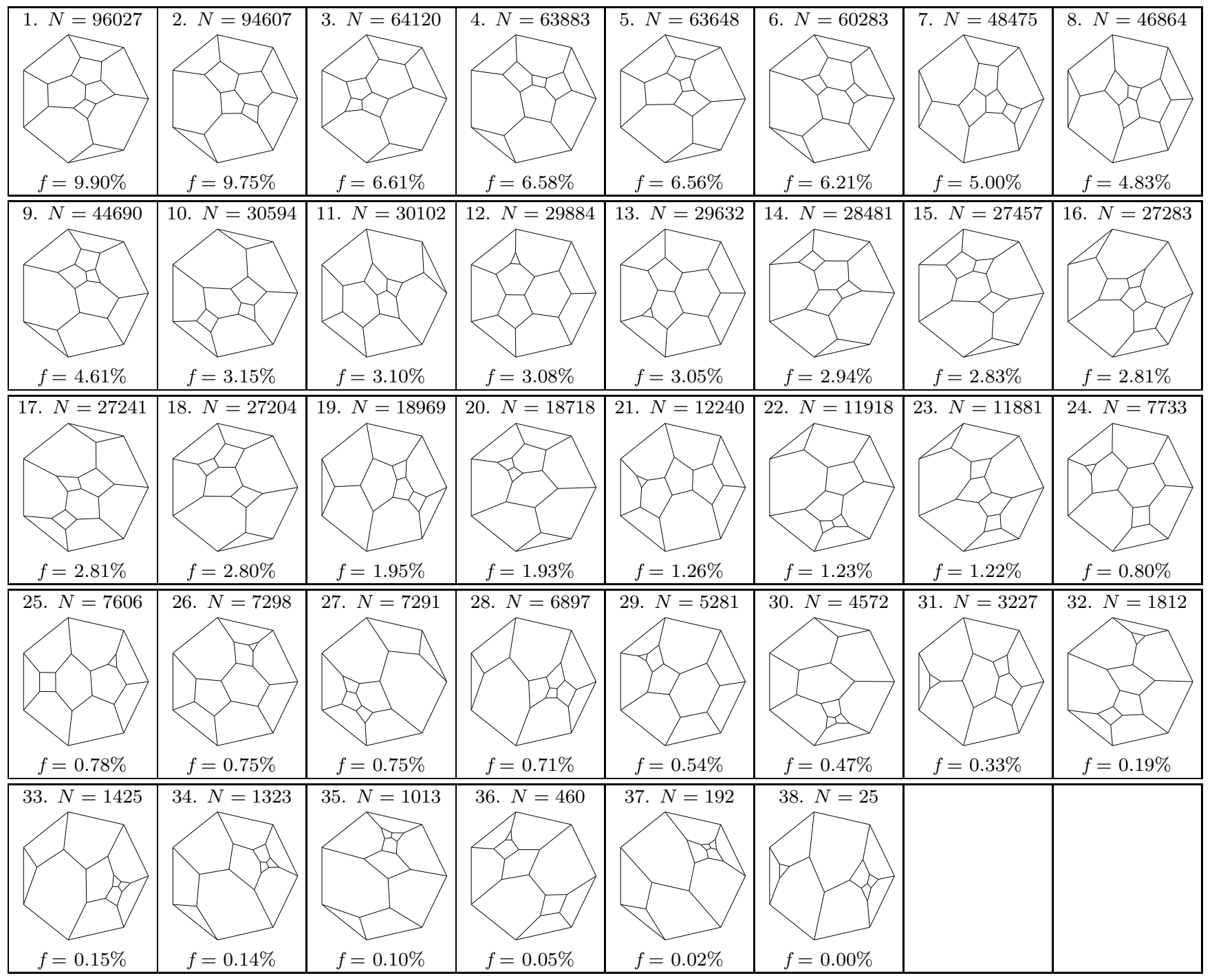

FIG. 20. Schlegel diagrams of all 38 topological types sharing $p$-vector $(001343100 \ldots)$. Listed for each type is its absolute frequency $N$ and the relative frequency $f$ among all types with the given $p$-vector. Entry 11 is the only one of these types which has a non-trivial symmetry $(S=2)$. 


\begin{tabular}{|r|c|r|r|}
\hline$F$ & $\alpha$ & $\beta$ & $\gamma$ \\
\hline 5 & $1.0516 \pm 0.1211$ & $26.8590 \pm 0.7646$ & $3.7451 \pm 0.3563$ \\
6 & $0.8235 \pm 0.0360$ & $23.9845 \pm 0.6768$ & $5.4220 \pm 0.2125$ \\
7 & $0.8658 \pm 0.0124$ & $21.2568 \pm 0.2521$ & $6.0360 \pm 0.0783$ \\
8 & $0.8499 \pm 0.0069$ & $20.3902 \pm 0.1754$ & $7.0576 \pm 0.0532$ \\
9 & $0.8519 \pm 0.0056$ & $19.4911 \pm 0.1576$ & $7.9790 \pm 0.0492$ \\
10 & $0.8585 \pm 0.0045$ & $18.6500 \pm 0.1362$ & $8.8564 \pm 0.0445$ \\
11 & $0.8593 \pm 0.0041$ & $18.1090 \pm 0.1306$ & $9.7961 \pm 0.0447$ \\
12 & $0.8658 \pm 0.0028$ & $17.4918 \pm 0.0910$ & $10.6799 \pm 0.0329$ \\
13 & $0.8740 \pm 0.0022$ & $16.8727 \pm 0.0731$ & $11.5388 \pm 0.0279$ \\
14 & $0.8766 \pm 0.0032$ & $16.4873 \pm 0.1106$ & $12.4630 \pm 0.0443$ \\
15 & $0.8816 \pm 0.0024$ & $16.0797 \pm 0.0856$ & $13.3712 \pm 0.0361$ \\
16 & $0.8813 \pm 0.0023$ & $15.8695 \pm 0.0826$ & $14.3440 \pm 0.0364$ \\
17 & $0.8869 \pm 0.0032$ & $15.4893 \pm 0.1186$ & $15.2387 \pm 0.0549$ \\
18 & $0.8855 \pm 0.0039$ & $15.3826 \pm 0.1474$ & $16.2469 \pm 0.0709$ \\
19 & $0.8952 \pm 0.0045$ & $14.8780 \pm 0.1683$ & $17.0569 \pm 0.0852$ \\
20 & $0.9004 \pm 0.0057$ & $14.5516 \pm 0.2130$ & $17.9413 \pm 0.1127$ \\
21 & $0.9038 \pm 0.0070$ & $14.3131 \pm 0.2653$ & $18.8698 \pm 0.1463$ \\
22 & $0.8952 \pm 0.0100$ & $14.5412 \pm 0.3981$ & $20.0455 \pm 0.2248$ \\
23 & $0.9304 \pm 0.0140$ & $13.1567 \pm 0.4973$ & $20.3107 \pm 0.3043$ \\
24 & $0.9087 \pm 0.0165$ & $13.8693 \pm 0.6431$ & $21.7840 \pm 0.3949$ \\
25 & $0.9553 \pm 0.0252$ & $12.0889 \pm 0.8403$ & $21.6639 \pm 0.5681$ \\
26 & $0.9459 \pm 0.0375$ & $12.2951 \pm 1.3040$ & $22.8030 \pm 0.8978$ \\
27 & $0.8497 \pm 0.0615$ & $16.3169 \pm 3.1440$ & $26.4506 \pm 1.9300$ \\
28 & $0.8858 \pm 0.0924$ & $14.7106 \pm 4.1820$ & $26.6911 \pm 2.7870$ \\
29 & $1.0230 \pm 0.1444$ & $9.7563 \pm 3.9500$ & $23.9996 \pm 3.3230$ \\
30 & $0.9302 \pm 0.2340$ & $12.4305 \pm 8.8750$ & $26.7969 \pm 6.6730$ \\
\hline
\end{tabular}

TABLE IX. Parameters for the partial distributions of cell volumes, as described in Section IV A.

\begin{tabular}{|r|c|c|r|}
\hline$F$ & $\alpha$ & $\beta$ & \multicolumn{1}{|c|}{$\gamma$} \\
\hline 5 & $1.5762 \pm 0.3972$ & $20.8402 \pm 1.4590$ & $6.3315 \pm 1.3440$ \\
6 & $1.2491 \pm 0.0495$ & $21.4514 \pm 0.7675$ & $9.2546 \pm 0.3419$ \\
7 & $1.3351 \pm 0.0231$ & $19.4808 \pm 0.3514$ & $10.2701 \pm 0.1670$ \\
8 & $1.3303 \pm 0.0130$ & $18.9765 \pm 0.2260$ & $11.8339 \pm 0.1099$ \\
9 & $1.3500 \pm 0.0093$ & $18.2157 \pm 0.1716$ & $13.1974 \pm 0.0877$ \\
10 & $1.3424 \pm 0.0045$ & $18.0385 \pm 0.0900$ & $14.7931 \pm 0.0478$ \\
11 & $1.3475 \pm 0.0054$ & $17.6702 \pm 0.1138$ & $16.2580 \pm 0.0634$ \\
12 & $1.3534 \pm 0.0051$ & $17.3403 \pm 0.1124$ & $17.7158 \pm 0.0657$ \\
13 & $1.3657 \pm 0.0043$ & $16.8931 \pm 0.0965$ & $19.0826 \pm 0.0594$ \\
14 & $1.3690 \pm 0.0034$ & $16.6523 \pm 0.0780$ & $20.5425 \pm 0.0502$ \\
15 & $1.3733 \pm 0.0045$ & $16.4274 \pm 0.1064$ & $22.0069 \pm 0.0715$ \\
16 & $1.3688 \pm 0.0057$ & $16.4174 \pm 0.1405$ & $23.6017 \pm 0.0980$ \\
17 & $1.3696 \pm 0.0056$ & $16.3018 \pm 0.1413$ & $25.1211 \pm 0.1024$ \\
18 & $1.3721 \pm 0.0049$ & $16.1507 \pm 0.1271$ & $26.6042 \pm 0.0957$ \\
19 & $1.3780 \pm 0.0062$ & $15.9226 \pm 0.1626$ & $28.0233 \pm 0.1273$ \\
20 & $1.3879 \pm 0.0085$ & $15.5909 \pm 0.2223$ & $29.3458 \pm 0.1814$ \\
21 & $1.4014 \pm 0.0096$ & $15.1868 \pm 0.2482$ & $30.6038 \pm 0.2116$ \\
22 & $1.3666 \pm 0.0114$ & $16.0624 \pm 0.3245$ & $32.9184 \pm 0.2772$ \\
23 & $1.4394 \pm 0.0195$ & $14.1189 \pm 0.4768$ & $32.8076 \pm 0.4465$ \\
24 & $1.3920 \pm 0.0291$ & $15.2752 \pm 0.8076$ & $35.4115 \pm 0.7474$ \\
25 & $1.4758 \pm 0.0386$ & $13.1021 \pm 0.8904$ & $34.8621 \pm 0.9126$ \\
26 & $1.4455 \pm 0.0612$ & $13.7373 \pm 1.5320$ & $37.0079 \pm 1.5720$ \\
27 & $1.2518 \pm 0.1002$ & $19.9525 \pm 4.1610$ & $44.3574 \pm 3.6360$ \\
28 & $1.3484 \pm 0.1052$ & $16.6244 \pm 3.4740$ & $43.2935 \pm 3.4210$ \\
29 & $1.4943 \pm 0.2177$ & $12.5015 \pm 5.0580$ & $40.4000 \pm 5.8690$ \\
30 & $1.4026 \pm 0.3032$ & $14.5465 \pm 8.7210$ & $43.7419 \pm 9.4830$ \\
\hline
\end{tabular}

TABLE X. Parameters for the partial distributions of cell surface areas, as described in Section IV B. 